\title{
Ezrin Prompted Myoblast Differentiation and Muscle Fiber Specialization and Gastrocnemius Muscle Repair in Peroneal Nerve Injury Model through PKA-NFATs Signaling Pathway
}

\section{Ruo-nan Zhang}

Hubei University of Medicine

Xin Bao

Hubei University of Medicine

Yan Wang

Hubei University of Medicine

Xin-Yuan Li

Hubei University of Medicine

MagdaleenaNaemi Mbadhi

Hubei University of Medicine

Yun Liu

Hubei University of Medicine

Wei Xu

Hubei University of Medicine

Lu-yuan Yao

Hubei University of Medicine

Long Chen

Hubei University of Medicine

Xiao-ying Zhao

Hubei University of Medicine

Chang-qing $\mathrm{Hu}$

Hubei University of Medicine

Jing-xuan Zhang

Hubei University of Medicine

Hong-tao Zheng

Hubei University of Medicine

Yan Wu

Hubei University of Medicine

Shi-You Chen

University of missouri 


\section{Shan Li}

Hubei University of Medicine

\section{Shao-juan Chen}

Hubei University of Medicine

Jing Lv

Hubei University of Medicine

Liu-liu Shi

Hubei University of Medicine

Jun-Ming Tang ( $\square$ tangjm416@163.com )

Hubei University of Medicine https://orcid.org/0000-0002-1591-7626

\section{Research}

Keywords: Ezrin, Differentiation, myoblast fusion, PKA, NFAT, MEF2c

Posted Date: November 10th, 2021

DOI: https://doi.org/10.21203/rs.3.rs-1038804/v1

License: (c) (1) This work is licensed under a Creative Commons Attribution 4.0 International License. Read Full License 


\section{Abstract}

Background: Muscular dystrophy is a destructive neuromuscular disease characterized by progressive muscle weakness and muscle atrophy. The role of Ezrin in myoblast differentiation/fusion and muscle atrophy is still unknown.

Method: Gastrocnemius muscle atrophy model were prepared by mechanical clamp of peroneal nerve. Differentiating $\mathrm{C} 2 \mathrm{C} 12$ cells treated with Ad-Ezrin or Ad-shEzrin were detected by gene chip, Q-PCR, immunofluorescence staining and Western blot.

Results: Ezrin was expressed in $\mathrm{MyHC} \mathrm{I/II} \mathrm{myofibers} \mathrm{in} \mathrm{vivo,} \mathrm{and} \mathrm{time-dependently} \mathrm{increased} \mathrm{during}$ myoblast differentiation/fusion characterized by MyoG+/MEF2c nuclei and MyHC+ myotubes in vitro. Overexpression of Ezrin promoted myoblast differentiation/fusion in time-dependent manner, inducing the increased $\mathrm{MyHC}-\mathrm{I}^{+}$and $\mathrm{MyHC}-\mathrm{II}^{+}$muscle fiber specialization, the specific effects could be abolished by addition of Ad-Periaxin. Ad-Ezrin did not alter PKA and PKAreg II a levels, but PKAreg I $a / \beta$. The PKA inhibitor, $\mathrm{H}-89$, remarkably abolished the over-expression effects by Ezrin on an increased myoblast differentiation/fusion. By contrast, Knockdown of Ezrin by shRNA significantly delayed myoblast differentiation/fusion accompanied by the decreased PKA reg I/II ratio, the inhibitory effects could be eliminated by PKAreg I activator N6-Bz-cAMP. Meanwhile, Ad-Ezrin enhanced type I muscle fiber specialization, accompanied by the increased levels of NFATc1/c2.Furthermore, Ad-NFATc2 or Ad-NFATc4 reversed the inhibitory effects of Ad-shEzrin on myoblast differentiation/fusion. Importantly, in vivo transfection of Ad-Ezrin into gastrocnemius muscles in peroneal nerve injury model increased the numbers of $\mathrm{MyHC}-\mathrm{I}^{+}$and $\mathrm{MyHC}-\mathrm{II}^{+}$myofibers, reducing muscle atrophy and fibrosis.

Conclusions: Ezrin activated PKA-NFAT-MyoD/MyoG/MEF2C signaling pathway, triggering myoblast differentiation/fusion and muscle fiber specialization in periaxin-depentdent manner, contributing to gastrocnemius muscles repair.

\section{Introduction}

Muscular dystrophy (MD) is a destructive neuromuscular disease characterized by progressive muscle weakness, muscle atrophy, and cardiac dysfunction ${ }^{1}$. In addition to dysfunction of neurotrophic effect, another possible contributor to the generation of pathology in MD is the primary muscular disorders ${ }^{2,3}$. Indeed, muscle satellite cells function in the physiological self-renewal and repair of pathological injury 4 . However, the underlying mechanism of muscle satellite cells remains unclear during the development of skeletal myopathy.

Charcot-Marie-Tooth (CMT), as a unique MD, characterized by hereditary motor sensory neuropathy ${ }^{5}$. Published data showed that Periaxin could be a candidate gene for CMT4F by destroying the myelin sheath formed by Schwann cells ${ }^{5}$. Of interest, Ezrin, a member of the Ezrin/radixin/moesin (ERM) protein family, is encoded byvillin2 located on chromosome 6q25.2-q26, which could inhibit the self-association 
of L-periaxin to participate in myelin sheath maintenance ${ }^{6}$. Evidence that the $\mathrm{N}$-terminal of Ezrin connecting with CD44 on the cell membrane ${ }^{7}$, and the C-terminal linking into actin filaments were involved in tumor invasion and metastasis indicated that Ezrin could play an important role in MD because CD44 and F-actin performed crucial function in muscle satellite cells differentiation and fusion.

Accumulated data showed that Ezrin could anchor cAMP dependent protein kinases, resulting in the activation of PKA, which phosphorylates enzyme proteins or channel proteins, such as $\mathrm{Na}+\mathrm{H}+$ exchanger (NHE3), and changes their gating properties ${ }^{8}$. Recent reports have shown that the changes of $\mathrm{pH}$ were closely related to the regeneration and repair of skeletal muscle mediated by satellite cells $\mathrm{s}^{9,10}$. Furthermore, the activated protein kinase A (PKA) has been linked to the unique phenomenon of myoblast differentiation/fusion and myotube formation, ascribing to the alteration in PKA regulatory subunit I (PKA $\mathrm{RI})$ under normal differentiation condition ${ }^{11}$. Our previous study suggested that it abated the ratio of PKA $\mathrm{RI} / \mathrm{RII}$ in myoblast cells, resulting in the postponement of myoblast differentiation and fusion ${ }^{12}$. Further evidence shows that ERM proteins act as PKA-anchoring proteins and sequester PKA close to its target proteins for their effective phosphorylation and functional regulation ${ }^{13}$. NFATs (Nuclear factor of activated T cells) activation mediated by PKA plays a crucial role in myoblast differentiation and fusion, myotube size, and altered muscle fiber specialization ${ }^{4,13}$. In this study, overexpression of Ezrin increased the number of MyHC-I/II myofibers, resulting in the recovery of gastrocnemius muscle atrophy in peroneal nerve injury model, which were attributed to the higher potential of myoblast cells differentiation/fusion and myofiber specialization through PKA-NFAT-MEF2C signaling pathway.

\section{Method}

\section{Animals, In vivo transfection and Peroneal nerve injury model preparement}

According to the Guide for the Care and Use of Laboratory Animals published by the US National Institutes of Health and China, animal studies were performed accordingly. Experimental Animal Centre of Hubei Medical University provided C57BL/6 mice (male, 3-5 months) that meet the criteria. The Institutional Animal Care and Use Committee of Hubei Medical University approved animal protocols.

For the transduction ofadult muscles, $\mathrm{C} 57 \mathrm{BL} / 6$ male micewere anesthetized by using an isoflurane vaporizer maintained at $2 \%$ isoflurane, $1 \mathrm{~L} / \mathrm{m}$ oxygen. Gastrocnemius and soleus (SL) muscles were exposed and injected with Ad-Ezrin ( $1 \times 10^{10} \mathrm{pfu}$, two point, $\left.50 \mu \mathrm{m} / \mathrm{each}\right)^{15}$. Muscles were removed 7 days after transfection, frozen inisopentane cooled in liquid nitrogen, and stored at $-80^{\circ} \mathrm{C}$. The efficiency of transfection was measured through detecting His-tag.

For gastrocnemius muscle atrophy observation, $\mathrm{C} 57 \mathrm{BL} / 6$ male mice were anesthetized by using an isoflurane vaporizer maintained at $2 \%$ isoflurane, $1 \mathrm{~L} / \mathrm{m}$ oxygen. Peroneal nerves were exposed and clamped for 15 minutes; subsequently, gastrocnemius muscle was injected with Ad-Ezrin, Ad-Periaxin 
and/or Ad-shPeriaxin $\left(1 \times 10^{10} \mathrm{pfu}\right.$, four point, $50 \mu \mathrm{m} /$ each $){ }^{15}$. Muscles were removed 14 days after transfection, frozen in isopentane cooled in liquid nitrogen, and stored at $-80^{\circ} \mathrm{C}$. The myofibers types were measured through double fluorescence immunostaining of MyHC-I (NOQ, ab234431) and MyHC-II (My32, ab51263). Meanwhile, Masson and hematoxylin-eosin staining was performed as following operation manual.

\section{C2C12 myoblast culture and differentiation induction}

C2C12 myoblast (Purchased from cell resource center of Shanghai Academy of life sciences, Chinese Academy of Sciences) were inoculated in $75-\mathrm{cm}^{2}$ culture dishesand cultured with proliferation medium (PM) containing high glucose DMEM (Gibco, USA, HG-DMEM) supplemented with 10\% FBS (C0225, AusGenex Fetal Bovine Serum Excellent ) at $37^{\circ} \mathrm{C}$ and $5 \% \mathrm{CO}_{2}$. When the confluence of the cells reached $75 \%$, the PM was replaced with differentiation medium (MD) containing HG-DMEM supplemented with $2 \%$ horse serum (HS, BI 04-124-1 A, Sigma, USA) to induce C2C12 myoblast cell differentiation. Traits of myotube formation from myoblast differentiation were observed daily under a microscope ${ }^{14}$.

\section{Adenoviral vector preparement and in vitro transfection}

Construction of Ezrin, L-Periaxin, NFATc1/c2 overexpression adenoviral vector were prepared as previously described ${ }^{15}$. Constructions of Ezrin, L-Periaxin and NFATc3/c4 short hairpin RNA (shRNA) adenoviral vector were prepared as previously described ${ }^{15}$. These overexpression adenoviral vectors containing Ad-NFATc1, Ad-NFATc2, Ad-shNFATc3 and Ad-shNFATc4 were obtained from Vigenebio. To confirm the role of L-Periaxin in myoblast, the addition of Ad-Null, Ad-Periaxin, or AdshPeriaxin $\left(1 \times 10^{9} \mathrm{pfu}\right)$ into corresponding culture dish one day before Ad-Ezrin or Ad-shEzrin was added. To confirm the role of NFATc3 or NFAtc4 on myoblast, the addition of Ad-Null, Ad-shNFATc3, or AdshNFATc4 $\left(1 \times 10^{9} \mathrm{pfu}\right)$ into corresponding culture dish one day before Ad-Ezrin or Ad-shEzrin was added. And then proliferation medium was replaced with differentiation medium for further observation.

\section{Immunofluorescence Staining}

C2C12 myoblast differentiation was determined by immunofluorescence staining. Primary monoclonal and polyclonal antibodies against MEF2C (\#5030s, 1:200, CST), MyHC (sc-20641, 1:150, Santa Cruze), MyHC-I (NOQ, ab234431,1:250, ABCAM) and MyHC-II (My32, ab51263,1:250, ABCAM) were added into each well in every group and incubated for $12 \mathrm{~h}$ at $4{ }^{\circ} \mathrm{C}$. The cells were washed with PBS 3 times for $15 \mathrm{~min}$ and incubated with appropriative fluorescent dye-labeled secondary antibodies (Jackson Lab, 1:500, USA) at $25^{\circ} \mathrm{C}$ for $2 \mathrm{~h}$. The nuclei were stained with DAPI (Molecular Probes). The images for each group were photographed under Nikon 80 i fluorescence microscope ${ }^{16}$. 


\section{Myoblast differentiation}

After myoblast were treated under DM for the indicated time, the differentiated myoblast was stained for MyoG or MEF2C using the primary polyclonal antibody MyoG (sc-12732, 1:150, Santa Cruze) or MEF2C (5030S, 1:400, CST) and appropriative TRITC-labeled secondary antibody (Jackson Lab, 1:500, USA). The nuclei were stained with DAPI. C2C12 myoblast with only 1-2 nuclei within a cellular structure were evaluated with MyoG or MEF2C staining. The MyoG+ or MEF2C+ cells were defined as the differentiated cells that did not fuse to form myotubes. Myoblast with 3 or more nucleiin the structure of a cell were defined as myotubes. The number of double-positive nuclei under high power field (HPF, $50 \mu \mathrm{m})$ were analyzed after double staining of MyoG/DAPI or MEF2C/DAPI. Two individuals who did not know the results evaluated the images using Image $\mathrm{J}$ (Java) software (National Institutes of Health, USA).

\section{Myoblast fusion and Myotube morphology}

The differentiated myoblast was stained for $\mathrm{MyHC}$ with the primary polyclonal antibody MyHC (rabbit anti-mice antibody, sc-20641, 1:150, Santa Cruze), MyHC-I (NOQ, ab234431, 1:250, ABCAM), MyHC-II (My32, ab51263, 1:250, ABCAM) and appropriative TRITC or FITC-labeled secondary antibody (Jackson Lab, 1:500, USA). C2C12 myoblast with only 1-2 nuclei within a cellular structure were evaluated by MyHC staining, indicating that the $\mathrm{MyHC}+$ cells were defined as the differentiated cells without mutually fusing to myotube. Myoblast with 3 or more nuclei in the structure of a cell were defined as myotube. The nuclei were stained with DAPI.

To analyze myotube morphology, we divided the cells into 2 groups, including short myotube with 3 5 myoblast fusion and long myotube with more than 5 myoblast fusions ${ }^{15,17}$. Two individuals who did not know the results evaluated the images using Image $\mathrm{J}$ (Java) software (National Institutes of Health, USA).

\section{Microarray analysis and Quantitative RT-PCR}

The $\mathrm{C} 2 \mathrm{C} 12$ myoblast treated with Ad-Ezrin or Ad-shEzrin for 6 days were prepared according to the manufacturer's protocol, and detected and analyzed (Shanghai OE Biotech CO, LTD). Total RNA from C2C12 myoblast was obtained using TRIzol (Invitrogen, Life Technologies) and transcribed into cDNA using the SuperScript II cDNA kit (Invitrogen, Life Technologies). Quantitative PCR was carried out using SYBR green PCR master mix (Thermo Fisher Scientific, Applied Biosystems, CN) in Real-Time PCR System (RotorGene 6000, Qiagen, Germany). The transcript levels of the gene of interest in each group were normalized to GAPDH levels ${ }^{18}$. The primers used are listed in Table 1.

Table1

The sequences of primers of qPCR. 


\begin{tabular}{lll} 
Gene & Forward & Reverse \\
\hline MyoG & 5'-GAGACATCCCCCTATTTCTACCA-3' & 5'-GCTCAGTCCGCTCATAGCC-3' \\
\hline MyoD1 & 5'-CCACTCCGGGACATAGACTTG-3' & 5'-AAAAGCGCAGGTCTGGTGAG-3' \\
\hline MyHC1 & 5'-CAAGCAGCAGTTGGATGAGCGACT-3' & 5'-TCCTCCAGCTCCTCGATGCGT-3' \\
\hline MyHC2a & 5'-AGAGGACGACTGCAGACCGAAT-3' & 5'-GAGTGAATGCTTGCTTCCCCCTTG-3' \\
\hline MyHC2b & 5'-ACGCTTGCACACAGAGTCAG-3' & 5'-CTTGGACTCTTCCTCTAGCTGCC-3' \\
\hline MyHC2x & 5'-ACCAAGGAGGAGGAACAGCAGC-3' & 5'-GAATGCCTGTTTGCCCCTGGAG-3' \\
\hline GAPDH & 5'-ATGACTCCACTCACGGCAAA-3' & 5'-ATGATGACCCTTTTGGCTCC-3' \\
\hline $\begin{array}{l}\text { qPCRs were performed to identified satellite cell differentiation and muscle fibers traits by using the } \\
\text { specific primers of satellite cell differentiation markers including MyoD and MyoG, type I muscle fiber } \\
\text { makers like MyHC1, and type II muscle fiber makers such as MyHC2a, MyHC2b, and MyHC2X. }\end{array}$
\end{tabular}

\section{Western blot}

C2C12 myoblastwere homogenized on ice in $0.1 \%$ Tween-20 homogenization buffer containing protease inhibitors. Nuclear and cytosolic proteins were separated and collected using NE-PER Nuclear and Cytoplasmic Extraction Reagents according to the manufacture's instruction (78835, Thermo Fisher Scientific, USA). $20 \mu \mathrm{g}$ of protein in each well were separated by 7 or $10 \%$ SDS-PAGE gel electrophoresis and transferred onto PVDF membrane (Millipore). After blocking with 5\% nonfat milk, the membranes were incubated with primary antibodies, including a-tubulin (T9026, 1:5000, Sigma), Histone H3 (ab6002, 1:500, ABCAM), MyoG (sc-12732, 1:250, Santa Cruze), MEF2C (5030S, 1:500, CST), NFATc1 (ab2796, 1:500, Abcam), NFATc2 (ab2722,1:500, Abcam), NFATc3(ab83832,1:500, Abcam),NFATc4 (SAB4501982, 1:1000, Sigma) and MyHC (sc-20641, sc-376157, 1:500, Santa Cruze) overnight at $4{ }^{\circ} \mathrm{C}$, respectively. Thereafter, the blots were incubated with corresponding horseradish peroxidase (HRP)-conjugated secondary antibodies (anti-rabbit IgG, anti-goat IgG, 1:10000; Santa Cruz) for 90 min. Protein expression was detected by enhanced chemiluminescence method, and the Image $\mathrm{J}$ software was used for gray value analysis ${ }^{19}$.

\section{Statistical analysis}

Data of quantitative and semi-quantitative analysis presented are mean \pm SD. Paired or unpaired Student's t-test determined statistical significance between the two groups. One-way ANOVA was used to compare the results for more than two experimental groups to specify the differences between groups. $P<0.05$ is considered meaningful.

\section{Results}




\section{Ezrin was specifically expressed in myoblasts and their skeletal muscle fibers}

To confirm the role of Ezrin in skeletal muscle, we firstly detected if Ezrin was expressed in gastrocnemius muscle, as shown in figure1A, myofibers partially were expressing Ezrin. To distinguish the traits of Ezrin expression in different myofibers including $\mathrm{MyHC}-\mathrm{I}$ and $\mathrm{MyHC}-\mathrm{Il}$, showing that more MyHC-Il myofibers were positive for Ezrin through double immunofluorescence staining, in addition to MyHC-I myofibers (Figure.1B-1D). Subsequently, to further confirm if Ezrin was expressed in myoblast cells and differentiating myoblast cells, the expression of Ezrin during the process of $\mathrm{C} 2 \mathrm{C} 12$ cells differentiation/fusion was analyzed by and immunofluorescence staining and western blotting. We found that Ezrin expression gradually increased during myoblast differentiation, reaching peak levels on day 4 of differentiation (Figure.1E-1G). Furthermore, in line with figure1B, differentiated C2C12 myoblast cells and formed myotubes showed the positive traits for Ezrin (Figure.1H). These results indicated that specific expression of Ezrin in myoblasts and their skeletal muscle fibers could involve in myoblast differentiation/fusion and muscle fiber specialization.

\section{Ezrin involved in myoblast differentiation/fusion}

To determine the effect of Ezrinon myoblast differentiation and fusion, we transfected $\mathrm{C} 2 \mathrm{C} 12$ cells with an adenovirus and shRNA to overexpress or knockdown the expression ofEzrin, respectively. Assessment of the transfection efficiency revealed that following the application of 100 optimal multiplication of infection (MOI) with the adenovirus, C2C12 myoblast almost reached a confluence of 95\%(sfig.1A-1C). His-tag was used to confirm the successful expression of the exogenous genes and their respective functions (sfig.1C-1E).

Our results further showed that the number of $\mathrm{MyHC}+$ myotubes with either 3-5 or $5^{+}$nuclei increased upon treatment with Ad-Ezrin in a time-dependent manner (Figure.2A-2D). Conversely, knockdown of Ezrin by shRNA did not only obviously reduce $\mathrm{MyHC}+$ cell number, but it also dramatically decreased myotube numbers with either 3-5 or $5^{+}$nuclei (Figure.2A-2D). Thisindicates that knockdown of Ezrin can almost effectively block myoblast differentiation and fusion. Combined with figure 1, these results demonstrated that Ezrin was involved in early differentiation and fusion of myoblast.

\section{MyoG/MEF2Cinvolved Ezrin-mediated myoblast differentiation and fusion}

MyoG and MEF2C have significant role, especially, during early and late myoblast differentiation ${ }^{20}$, we used gene chip to analyze the traits of myoblast differentiation related genes in differentiated myoblast cells treated with Ad-Ezrin or Ad-shEzrin, As shown in Figure. 2E, the expression of myoblast differentiation-associated genes,includingMyf5, Myf6, MyoD, MyoG, MEF2a, MEF2b,MEF2c and MEF2d 
were analyzed (Figure. 2E). We then performed MyoG and MEF2C immunostaining assay to explore the relationship between Ezrin and the myogenic markers. Our results showed that following treatment with Ad-Ezrin, the MyoG and MEF2C expression increased, with the number of $\mathrm{MyoG}^{+}$nuclei significantly higher than that of MEF2C+ nuclei (Figure.2F-2I). Meanwhile, the knockdown effects of Ezrin with shRNA resulted in markedly declined number of MyoG+ and MEF2C+ nuclei cells in differentiated myoblast cells (Figure.2F-2I). In a word, these results indicated that Ezrin affects myoblast differentiation via the modulation of myogenic markers, MyoG and MEF2C, but more especially, MyoG.

\section{Ezrin involved in myofiber specialization}

Because skeletal muscle function differences were associated with muscle fibers types ${ }^{14}$, we used gene chip to analyze the traits of myofibers types in differentiated myoblast cells treated with Ad-Ezrin or AdshEzrin. As shown in Figure. 3A, the expression of myofibers type-associated genes,includingMyHC-1, MyHC-2a, MyHC-2b, MyHC-2X, Myh3, Myh4, Myh6, Myh7b, Myh9, Myh10 and Myh13 were analyzed (Figure.3A).The results showed that overexpressing-Ezrin obviously increased both 'fast muscle' MyHC-II and 'slow muscle'MyHC-I positive myotube formation(Figure. 3B-3E). Conversely, knockdown of Ezrin by shRNA slightly increased MyHC-II positive myotube numbers while it reduced MyHC-I positive myotube numbers (Figure. 3B-3E), like Q-PCR' results as shown in sFigure2. To further confirm the effects of Ezrin on muscle fiber type composition, we investigated the role of overexpressing-Ezrin in the gastrocnemius (GA) and soleus (SL) muscles. The results showed that MyHC type I fibers were significantly increased in both muscles (Figure.3F-3I). These results show that Ezrin regulates the various $\mathrm{MyHC}$ isoforms in vivo and in vitro.

\section{Interaction between Ezrin and L-Periaxin involved in myoblast differentiation and myofiber specialization}

Since mutation and deletion of L-Periaxin was associated with Charcot-Marie-Tooth (CMT) characterized by progressive muscle weakness and atrophy of distal extremities with sensory impairment through destroying the myelin sheath formed by Schwann cells $s^{4,5}$. Interestingly, Ezrin could inhibit the selfassociation of L-periaxin to participate in myelin sheath maintenance. These results push us to explore if Ezrin-mediated myoblast differentiation involved in L-Periaxin, we firstly found that L-Periaxin expression were gradually increased and reached the peak at 6 day of myoblast differentiation (sFigure3A-3C), which was different from the peak expression of Ezrin at 4 day of myoblast differentiation (Figure1F), indicating that Ezrin could affect myoblast differentiation through regulating L-periaxin. Unfortunately, as shown in Figure 4A-4E and sFigure4, overexpression of alone L-Periaxin (Ad-Periaxin) did not alter Ezrin-induced myoblast differentiation, but overexpression of L-Periaxin obviously abolished the beneficial effect of overexpressing-Ezrin on myoblast differentiation while knockdown of L-Periaxin by shRNA slightly enhanced overexpressing-Ezrin' effects. These results indicated that interaction between Ezrin and LPeriaxin involved in myoblast differentiation, at least in part. 
To further confirm if Ezrin and L-Periaxin involved in myoblast differentiation and myofiber specialization, we established peroneal nerve injury (PNI) model to partially mimic CMT-associated muscle atrophy, results showed the traits of gastrocnemius muscle atrophy (sFigure.5). Meanwhile, we found that overexpression of Ezrin markedly increased $\mathrm{MyHC}-\mathrm{I}$ and $\mathrm{MyHC}-\mathrm{Il}$ positive myofibers numbers (Figure5A5D) while it reduced muscle fibrosis (sFigure.5). Unfortunately, overexpression of L-Periaxin (Ad-Periaxin) did not recover the PNI-induced gastrocnemius muscle atrophy but deteriorated its fibrosis (sFigure.5). Furthermore, knockdown of L-Periaxin by shRNA could not enhance the beneficial effects of Ad-Ezrin on repairing gastrocnemius muscle injury mediated by PNI. Of interest, Ad-Periaxin reduced the numbers of MyHC-II weakly positive myofibers compared with sham and PNI groups. And knockdown of L-Periaxin by shRNA did not abolish the enhanced effects of Ezrin on MyHC-II weakly positive myofibers (Figure.5A5D). These results suggested that myoblast differentiation and myofiber specialization did not directly involve in L-Periaxin, but Ezrin.

\section{Ezrin regulated myoblast differentiation and fusion through PKA signaling pathway}

Previous studies have shown that the PKA and PKAreg I/II ratio play crucial roles in controlling myoblast differentiation and fusion ${ }^{11,12}$. To confirm if Ezrin's role in myoblast differentiation and fusion was involved in PKA signaling pathway, we used gene chip and western blot to analyze the traits of PKA signaling pathwayin differentiated myoblast cells treated with Ad-Ezrin or Ad-shEzrin. Our results revealed that, the overexpression of Ezrin did not alter PKAreg II levels, but it significantly increased the levels of PKAa, PKAreg la, and PKAreg I $\beta$, resulting in an increased PKAreg $\mathrm{I} / \mathrm{II}$ ratio. By contrast, knockdown of Ezrin by shRNA significantly reduced PKAreg la and PKAreg I $\beta$ levels, but it did not alter PKAreg II levels, resulting in a decreased PKAreg $\mathrm{I} / \mathrm{II}$ ratio (Figure.6A-6H).

Since the activity of PKA has been reported to have effects on myoblast differentiation and fusion ${ }^{11,12}$. Combining with the above observations that the overexpression or knockdown of Ezrin affected myoblast differentiation and fusion by altering PKA activity, we treated $\mathrm{C} 2 \mathrm{C} 12$ cells with a PKA inhibitor $(\mathrm{H}-89)$. We found that the inhibition of PKA abolished the beneficial effects of Ezrin onmyoblast differentiation and fusion. By contrast, the PKA activator reversed the inhibitory effects of Ezrin knockdown on $\mathrm{C} 2 \mathrm{C} 12$ myoblast differentiation and fusion (Figure.61-6K). These results indicated that Ezrin mediation of myoblast differentiation and fusion is associated with the PKA signaling pathway.

\section{Ezrin regulated myoblast differentiation and fusion through PKA-MyoG/MEF2C signaling pathway}

Indeed, myotube is formed by the fusion of differentiated myoblasts, which is characterized by three $\left(3^{+}\right)$ or morenuclei in the cell structure ${ }^{15,17}$. As shown in Figure. 6I and sFigure.6-7, we found that knockdown of Ezrin by shRNA markedly decreased $\mathrm{MyoG}^{+}$and MEF2C ${ }^{+}$less than 3 nuclei, and these effects could be 
abolished by PKA activator. By contrast, overexpression of Ezrin substantially increased the number of MyoG+ or MEF2C+ nuclei in less than 3 and $3^{+}$myotubes. Furthermore, western blot showed that overexpression of Ezrin increased the nuclear levels of MyoG and MEF2C while knockdown of Ezrin by shRNA reduced it. More important, these specific changes could be cancelled by the PKA inhibitor and PKA activator, respectively (sFigure.6-7). These results indicated that Ezrin participated in $\mathrm{C} 2 \mathrm{C} 12$ myoblast differentiation and fusion through PKA-MyoG/MEF2C signaling pathway.

\section{NFATs signaling involved in the regulation of myoblast differentiation/fusionmediated by Ezrin}

Since NFATs play a crucial important role in myoblast differentiation/fusion, especially myotube

specification ${ }^{26-32}$. We used gene chip and western blot to analyze the traits of NFATs signaling pathway in differentiated myoblast cells treated with Ad-Ezrin or Ad-shEzrin. Results showed that the overexpression of Ezrin promoted nuclear translocation of NFATc1/c2 that led to increased levels of NFATc1/c2 nuclei, and decreased NFATc3/c4 nuclei level. Conversely, knocking down Ezrin induced nuclear translocation of NFATc3/c4 resulting in increased NFATC3/c4 nuclei level, and decreased the NFATc1/c2 nuclei levels (Figure.7A-7G, sFigure8). Secondly, the overexpression of NFATc2 or knockdown of NFATc4 almost completely reversed the inhibitory effects of knocking-down Ezrin during myoblast differentiation and fusion, resulting in the recovery of slim-long myotubes (nuclei numbers more than 5 ) (Figure.7F-7G). Eventually, NFATc1/c3 knockdown did not significantly recuperate the inhibitory effects of knocking-down Ezrin by shRNA in myoblast differentiation/fusion (Figure.7F-7G).

Since MyoG and MEF2C are involved in the initiation and later stage of myoblast differentiation, respectively ${ }^{26}$. As shown in Figure.7F-7GandsFigure. 9, we found that knockdown of Ezrin by shRNA markedly decreased the number and percentage of MyoG+ and MEF2C+ nuclei in less than 3-nuclei cells and 3-nuclei ${ }^{+}$myotubes, and treatment with Ad-NFATc2 or Ad-shNFATc4 could abolish these effects. In addition, Ad-NFATc1 or Ad-shNFATc3 reversed the number of MEF2C+ nuclei in 3-nuclei ${ }^{+}$myotubes. These results indicated that Ezrin participated in $\mathrm{C} 2 \mathrm{C} 12$ myoblast differentiation and fusion with coordination of MyoG and MEF2C, which were associated with NFATc2/c4, at least in part.

\section{NFATs signaling involved in the regulation of myofiber specialization mediated by Ezrin}

Previous studies have reported on two types of muscle fibers, including slow (slim-long) and fast (thickshort) myotubes, MyHC-I forms the former, and MyHC-2a, MyHC-2b or MyHC-2X the latter ${ }^{24,25}$.As shown in Figure. 8A-8D, we found that overexpression of Ezrin increased the numbers of MyHC-I and MyHC-II positive myotubes while knockdown of Ezrin decreased it, the specific effects could be abolished by AdNFATc1/c2 or Ad-shNFATc3/c4. More importantly, the specific increase in MyHC-2a and MyHC-2b mediated by knockdown of Ezrin could be obviously inversed by Ad-NFATc1/c2 or Ad-shNFATc4 
(sFigure.10), respectively. Thus, Ezrin mainly regulated myofiber specification through integration role of NFATs signaling pathway.

\section{Discussion}

In this study, we made three novel observations. Firstly, we found that the Ezrin was expressed in $\mathrm{MyHC}-\mathrm{I}^{+}$ and $\mathrm{MyHC}-\mathrm{II}^{+}$myofibers and showing a time-dynamic expression traits during myoblast differentiation and fusion. Secondly, Ezrin significantly controlled myoblast differentiation and fusion, which were related with PKA RI-NFAT-MyoD/MEF2C signaling pathway. And lastly, Ezrin contributed to the regeneration and repair of damaged gastrocnemius muscle induced by peroneal nerve injury.

Clinically, exploring the potential target of gene therapy for muscle atrophy is a hot spot. As one of ERM proteins, Ezrin activated the intracellular signal pathways through transferring extracellular signal molecules into actin cytoskeleton, affecting several key cellular processes, including membrane dynamics, cell adhesion, cell survival, motility, and determination of cell shape ${ }^{6-10}$. Indeed, these cellular processes were associated with myoblast differentiation and fusion ${ }^{21}$. Herein, for the first time, we found that $\mathrm{MyHC}-\mathrm{I}^{+}$and $\mathrm{MyHC}-\mathrm{II}^{+}$myofibers showed the positive expression of Ezrin, and its levels gradually increased during myoblast differentiation/fusion, and rapidly decreased when myotubes approached and/or reached maturity. Although Periaxin could be a candidate gene for CMT4F by destroying the myelin sheath formed by Schwann cells ${ }^{5}$. Our novel finding further showed that overexpression of Lperiaxin (Ad-periaxin) obviously abolished the effect of Ezrin on both myoblast differentiation/fusion in vitro and muscle injury repair in vivo. Unfortunately, knockdown of L-periaxin by shRNA did not markedly enhance the therapeutic effect of Ezrin on muscle injury. Considering the recent report that Ezrin could inhibit the self-association of L-periaxin to participate in myelin sheath maintenance ${ }^{6}$, we conjectured that not L-periaxin, but Ezrin was beneficial way of gene therapy for the injured muscles including CMT4F.

Muscle stem/progenitor cells including myoblast cells played a crucial role in skeletal muscle regeneration and restoration in physiological and pathological conditions. Previous studies have shown that the overexpression of PKA inhibited myogenic differentiation, contributing to HDAC4 phosphorylation and the transcriptional repression of muscle-specific genes by the myogenic regulators Myf-5 and MyoD 22, 23, 24, and 25. Furthermore, ERM proteins, including Ezrin, act as PKA-anchoring proteins and sequester PKA close to its target proteins for their effective phosphorylation and functional regulation ${ }^{13}$. However, our results did not only show any apparent changes in expressions of Myf- 5 and MyoD during the overexpression of Ezrin in myoblast but also showed the increased number of MyoG and MEF2C-positive myoblast cells and myotubes. MyoG and MEF2C play an essential role in the initiation and later stage of myoblast differentiation, respectively ${ }^{26}$. Recent reports have also associated the involvement of PKA RI and RII during myoblast differentiation and myotube formation ${ }^{11,12}$. Of interest, we found that overexpressingEzrin markedly increased PKA RI levels, leading to an increased PKA RI/RII ratio, accompanied by an acceleration of myoblast differentiation and fusion. Furthermore, the knockdown of Ezrin, resulting in a lower PKA RI/RII ratio, inhibitory effects observed during myoblast 
differentiation/fusion could be reversed by the PKA RI activator. More importantly, PKA RI activator almost completely recovered the number of MyoG or MEF2C positive myotubes. Therefore, Ezrin could promote myoblast differentiation/fusion via PKA RI/RII-MyoG/MEF2C signaling pathway.

Existing data have shown that NFATs are crucial players in myoblast differentiation and fusion, especially myotube specification ${ }^{26-32}$. Moreover, NFATs activities are frequently regulated by the PKAcalcineurinsignaling pathway during cell differentiation $33,34,35$, and 36 . Indeed, NFATc2 primarily controlled myoblast recruitment and myoblast fusion $37,38,39,40$. Similarly, we found that the overexpression of NFATc2 could restore the inhibitory effect of myoblast fusion by knockdown of Ezrin, but not NFATc1. In line with stimulatory effect of constitutively active NFATc1 (caNFATc1) on MyHC-1 expresison ${ }^{41}$, the increased NFATc1 levels by overexpression of Ezrin induced MyHC-1 expression. However, enhanced NFATc1 levels by Ad-Ezrin did not decrease MyHC-2b as caNFATc1 but maintained its normal levels. Meanwhile, treatment with Ad-Ezrin reduced NFATc3/c4 levels in myoblast, but maintained MyHC-2a expression, which was different from the inhibitory role of knockdown for NFATc3 and NFATc4 in the expression of $\mathrm{MyHC}-2 \mathrm{a}^{30}$. These different changes could be related to the increased levels of NFATc2 by Ad-Ezrin, because NFATc2 played an important role in MyHC-2a and MyHC-2X expressions ${ }^{37,40,41}$. In a word, altered NFATs signaling by Ezrin affected myoblast differentiation/fusion, especially type- 1 and type-2a muscle fiber specialization.

Concerning muscle fiber type specialization, slow muscle specialization and fast to slow myotube-type switch need the coordination of MEF2C and MyoD $39,40,41,42$, and 43 , respectively. In this study, the overexpression of Ezrin increased the MEF2C levels within the nucleus while decreasing the levels of MyoD in myoblast, resulting in the increased MyHC-1 expressions, accompanied by normal levels of MyHC-2a, MyHC-2b and MyHC-2X. The knockdown of Ezrin reduced MEF2C+ myoblast numbers while increasing MyoD expressions, leading to special changes that the levels MyHC-2a and MyHC-2b were obviously increased. Previous reports have shown that NFATc2 had synergistic effects with MyoD to activate the expression of MyHC-2a and MyHC-2X, while NFATc1 promoted MyHC-1 expression through

inhibiting MyoD signaling ${ }^{37,40}$. More, NFATc4 has shown the inhibitory role in myoblast fusion ${ }^{38}$, and appears to mostly contribute to fast muscle fiber formation, especially MyHC- $2 b^{30}$. The overexpression of NFATc4 affects the differentiation process by decreasing the expression of late differentiation markers, including MEF2c, and impairs myotube formation ${ }^{30,38}$. We found that these similar effects mediated by the knockdown of Ezrin could be reversed by knockdown of NFATc4. Thus, Ezrin could regulate myoblast fusion and type Ilb muscle fiber specialization through NFATc4-MEF2C signaling pathway.

\section{Conclusion}

Ezrin activated PKA-NFAT-MyoD/MyoG/MEF2C signaling pathway, triggering myoblast differentiation/fusion and muscle fiber specialization in Periaxin-depentdent manner, contributing to gastrocnemius muscles repair. 


\section{Abbreviations}

DAPI: 4',6-Diamidino-2-Phenylindole; DMEM: Dulbecco's Modified Eagle's Medium; FBS: Fetal Bovine Serum; HF: heart failure; HRP: horseradish peroxidase; HS: horse serum; IF: Immunofluorescence; MD: Muscular dystrophy; MyHC: myosin heavy chain; NFAT:nuclear factor of activated T cells; MEF2C:Myocyte-specific enhancer factor 2C; MyoD: myogenic differentiation 1; M: Mol/L; PKA: protein kinase A; PKA RIa: PKA regulatory subunit la; PVDF: polyvinylidene fluoride.

\section{Declarations}

\section{Ethics approval and consent to participate}

Not applicable.

\section{Competing interests}

The authors declare that they have no competing interests.

\section{Availability of Data and material}

Please contact corresponding author for data requests.

\section{Consent for publication}

Not applicable.

\section{Author contributions}

R.L.Z, X.B, Y.W mainly performed cells experimental and prepared the first draft; W.X and C.Q.H fulfilled qPCR; R.N.Z, Y.W and H.T.Z performed protein detection; X.Y.L and Y.W participated in immunostaining; X.Y Z, M.M.N and Y.L participated in myotube analysis; L.Y.Y and L.C participated in Adenovirus preparation; J.X.Z and J.L had a hand in the study design; M.N.M and S.Y.C partook in revising the manuscript; J.L, L.L.S and J.M.T composed the study, partook in the design and coordination of the whole study, and was conducive to revising the manuscript. All authors have read and agreed to the final draft.

\section{Funding}


The present study was supported by projects from the Foundation of Hubei Science\& Technology Department (2018ACA162 to J.M.T), Hubei Province's Outstanding Medical Academic Leader program, the Foundation of Hubei University of Medicine (HBMUPI201807, FDFR201601 to J.M.T), the National Natural Science Foundation of China (81670272 to J.M.T), Health Commission of Hubei Province scientific research project (WJ2019M051).

\section{Acknowledgements}

Not applicable

\section{References}

1. Smith SA, Downey RM, Williamson JW, Mizuno M. Autonomic dysfunction in muscular dystrophy: a theoretical framework for muscle reflex involvement. Front Physiol. 2014; 5:47.

2. Kowalski K, Dos Santos M, Maire P, Ciemerych MA, BrzoskaE.Induction of bone marrow-derived cells myogenic identity by their interactions with the satellite cell niche.Stem Cell Res Ther. 2018;9(1):258.

3. Sun C, Serra C, Lee G, Wagner KR.Stem cell-based therapies for Duchenne muscular dystrophy.Exp Neurol. 2020;323:113086.

4. Yin H, Price F, Rudnicki MA. Satellite cells and the muscle stem cell niche.Physiol Rev. 2013;93(1):23-67.

5. Williams A.C. Brophy p.J. The function of the Periaxin gene during nerve repair in a model of CMT4F. Journal of Anatomy. 2002; 200(4):323-330.

6. Guo T, Zhang L, Xiao H, Yang Y, Shi YW. Ezrin interacts with L-periaxin by the "head to head and tail to tail" mode and influences the location of L-periaxin in Schwann cell RSC96. BBA-General Subjects. 2020; 1864(4):129520.

7. Pore D, Gupta N.Ezrin-radixin-moesin family proteins in the regulation of B cell immune response. Crit Rev Immunol. 2015,35:15-31.

8. Bretscher A, Edwards K, Fehon RG. ERM proteins and merlin: integrators at the cell cortex. Nat Rev Mol Cell Biol. 2002; 3:586-599.

9. Arpin M, Chirivino D, Naba A, Zwaenepoell.Emerging role for ERM proteins in cell adhesion and migration. Cell AdhesMigr.2011;5:199-206.

10. Kishore R, Qin G, Luedemann C, Bord E, Hanley A, Silver M, Gavin M, Yoon YS, Goukassian D, Losordo DW. The cytoskeletal protein ezrin regulates EC proliferation and angiogenesis via TNF-alphainduced transcriptional repression of cyclin A. J Clin Invest. 2005;115(7):1785-96.

11. Han SY, Park DY, Lee GH, Park SD, Hong SH. Involvement of type I protein kinase A in the differentiation of L6 myoblast in conjunction with phosphatidylinositol 3-kinase. Mol Cells. 2002;14(1):68-74. 
12. Chen SJ, Yue J, Zhang JX, Jiang M, Hu TQ, Leng WD, Xiang L, Li XY, Zhang L, Zheng F, Yuan Y, Guo LY, Pan YM, Yan YW, Wang JN, Chen SY, Tang JM.Continuous exposure of isoprenaline inhibits myoblast differentiation and fusion through PKA/ERK1/2-FOX01 signaling pathway. Stem Cell Res Ther.2019;10:70.

13. Kawaguchi K, Yoshida S, Hatano R, et al. Pathophysiological Roles of Ezrin/Radixin/Moesin Proteins. Biological \& Pharmaceutical Bulletin, 2017, 40(4):381-390.

14. Chen SJ, Xiang L, Jiang M, et al. Isoprenaline induced muscle atrophy by inhibiting C2C12 cell differentiation into skeletal muscle cells. Chin J Cell Biol. 2017; 39:1178-1187.

15. Hicks MR, Cao TV, StandleyPR. Biomechanical strain vehicles for fibroblast-directed skeletal myoblast differentiation and myotube functionality in a novel coculture. Am J Physiol Cell Physiol. 2014; 307:C671-83.

16. Ross JA, Levy Y, Svensson K, et al.SIRT1 regulates nuclear number and domain size in skeletal muscle fibers. J Cell Physiol. 2018, 233:7157-716.

17. Horsley V, Jansen KM, Mills ST, Pavlath GK. IL-4 acts as a myoblast recruitment factor during mammalian muscle growth.Cell. 2003;113:483-94.

18. Chen X, Wan J, Yu B, et al.PIP5K1 a promotes myogenic differentiation via AKT activation and calcium release. Stem Cell Res Ther. 2018; 9(1):33.

19. Li GH, Luo B, Lv YX, et al.Dual effects of VEGF-B on activating cardiomyocytesand cardiac stem cells to protect the heart against short- and long-term ischemia-reperfusion injury.JTrans/ Med. 2016; 14:116.

20. Cheng X, Du J, Shen L, Tan Z, Jiang D, Jiang A, Li Q, Tang G, Jiang Y, Wang J, Li X, Zhang S, Zhu L. MiR-204-5p regulates C2C12 myoblast differentiation by targeting MEF2C and ERRY. Biomed Pharmacother. 2018; 101:528-535.

21. Palmer S, Groves N, Schindeler A, Yeoh T, Biben C, Wang CC, Sparrow DB, Barnett L, Jenkins NA, Copeland NG, Koentgen F, Mohun T, Harvey RP. The small muscle-specific protein CsI modifies cell shape and promotes myocyte fusion in an insulin-like growth factor 1-dependent manner. J Cell Biol. 2001;153(5):985-98.

22. Hardy S, Kong Y, Konieczny SF. Fibroblast growth factor inhibits MRF4activity independently of the phosphorylation status of a conserved threonineresidue within the DNA-binding domain. Mol Cell Biol. 1993;13(10):5943-56.

23. Winter B, Braun T, Arnold HH. cAMP-dependent protein kinase represses myogenic differentiation and the activity of the muscle-specific helix-loop-helix transcription factors Myf-5 and MyoD. J Biol Chem. 1993;268(13):9869-78.

24. Doddi SK, Kummari G, M V J, Kalle AM. Protein kinase A mediates novel serine-584 phosphorylation of HDAC4. Biochem Cell Biol. 2019;97(5):526-535.

25. Arnold HH, Braun T, Bober E, Buchberger A, Winter B, Salminen A. Regulationofmyogenin expression in normal and transformed myogenic cell lines. SympSocExp Biol. 1992;46:37-51. 
26. Cho YY, Yao K, Bode AM, Bergen HR 3rd, Madden BJ, Oh SM, Ermakova S, Kang BS, Choi HS, Shim $\mathrm{JH}$, Dong Z.RSK2 mediates muscle cell differentiation through regulation of NFAT3.J Biol Chem. 2007;282:8380-92.

27. O'Connor R S,Mills S T, Jones K A, et al. A combinatorial role for NFAT5 in both myoblast migration and differentiation during skeletal muscle myogenesis. Journal of Cell Science. 2007. 120:149-159.

28. Kegley KM, Gephart J, Warren GL, PavlathGK.Altered primary myogenesis in NFATC3(-/-) mice leads to decreased muscle size in the adult.Dev Biol. 2001;232:115-26.

29. Xu M, Chen X, Chen D, Yu B, Li M, He J, Huang Z.MicroRNA-499-5p regulates skeletal myotube specification via NFATc1/MEF2C pathway and Thrap1/MEF2C axis.Life Sci. 2018;215:236-245.

30. Calabria E, Ciciliot S, Moretti I, Garcia M, Picard A, Dyar KA, Pallafacchina G, Tothova J, Schiaffino S, MurgiaM.NFAT isoforms control activity-dependent muscle fiber type specification.Proc Natl AcadSci U S A. 2009;106:13335-40.

31. Du J, Shen L, Zhang P, Tan Z, Cheng X, Luo J, Zhao X, Yang Q, Gu H, Jiang A,Ma J, Tang Q, Jin L, Shuai S, Li M, Jiang Y, Tang G, Bai L, Li X, Wang J, ZhangS, Zhu L. The regulation of skeletal muscle fiber-type composition by betaine is associated with NFATc1/MyoD. J Mol Med (Berl). 2018;96:685700.

32. Ehlers ML, Celona B, Black BL.NFATc1 controls skeletal muscle fiber type and is a negative regulator of MyoDactivity.Cell Rep. 2014;8:1639-1648.

33. Khalilimeybodi A, Daneshmehr A, Sharif KashaniB.Ca2+-dependent calcineurin/NFAT signaling in $\beta$ adrenergic-induced cardiac hypertrophy. GenPhysiolBiophys. 2018;37:41-56.

34. Horsley V, PavlathGK.NFAT: ubiquitous regulator of cell differentiation and adaptation.J Cell Biol. 2002;156:771-4.

35. Swoap SJ, Hunter RB, Stevenson EJ, Felton HM, Kansagra NV, Lang JM, Esser KA, KandarianSC.Thecalcineurin-NFAT pathway and muscle fiber-type gene expression.Am J Physiol Cell Physiol. 2000;279:C915-24.

36. Daou N, Lecolle S, Lefebvre S, dellaGaspera B, Charbonnier F, Chanoine C, Armand AS.A new role for the calcineurin/NFAT pathway in neonatal myosin heavy chain expression via the NFATc2/MyoD complex during mouse myogenesis.Development. 2013;140:4914-25.

37. Horsley V, Friday BB, Matteson S, Kegley KM, Gephart J, PavlathGK.Regulation of the growth of multinucleated muscle cells by an NFATC2-dependent pathway.J Cell Biol. 2001;153:329-38.

38. Perroud J, Bernheim L, Frieden M, Koenig S.Distinct roles of NFATc1 and NFATc4 in human primary myoblast differentiation and in the maintenance of reserve cells. J Cell Sci. 2017;130:3083-3093.

39. Horsley V, Jansen KM, Mills ST, Pavlath GK IL-4 acts as a myoblast recruitment factor during mammalian muscle growth.Cell. 2003;113:483-94.

40. Daou N, Lecolle S, Lefebvre S, dellaGaspera B, Charbonnier F, Chanoine C, Armand AS.A new role for the calcineurin/NFAT pathway in neonatal myosin heavy chain expression via the NFATc2/MyoD complex during mouse myogenesis.Development. 2013;140:4914-25. 
41. McCullagh KJ, Calabria E, Pallafacchina G, Ciciliot S, Serrano AL, Argentini C, Kalhovde JM, Lømo T, Schiaffino S.NFAT is a nerve activity sensor in skeletal muscle and controls activity-dependent myosin switching. Proc Natl AcadSci US A. 2004;101:10590-5.

42. Melissa L, Ehlers, Barbara. NFATc1 Controls Skeletal Muscle Fiber Type and Is a Negative Regulator of MyoD Activity. Cell Reports. 2014;8:1639-1648.

43. Koo JH, Kim TH, Park SY, Joo MS, Han CY, Choi CS, Kim SG.Ga13 ablation reprograms myotubes to oxidative phenotype and enhances whole-body metabolism.JClin Invest. 2017;127:3845-3860.

\section{Figures}

A

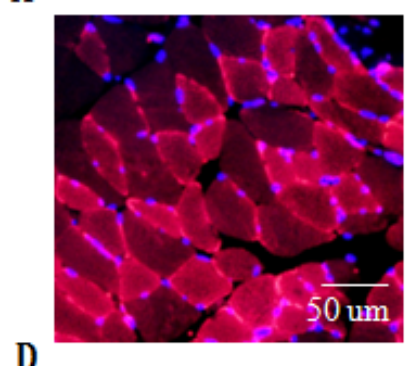

D

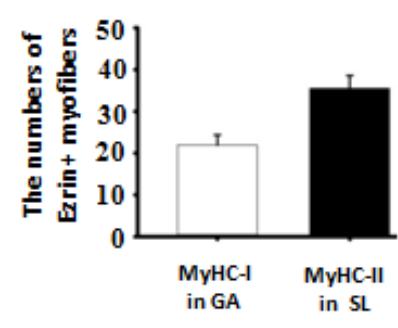

E
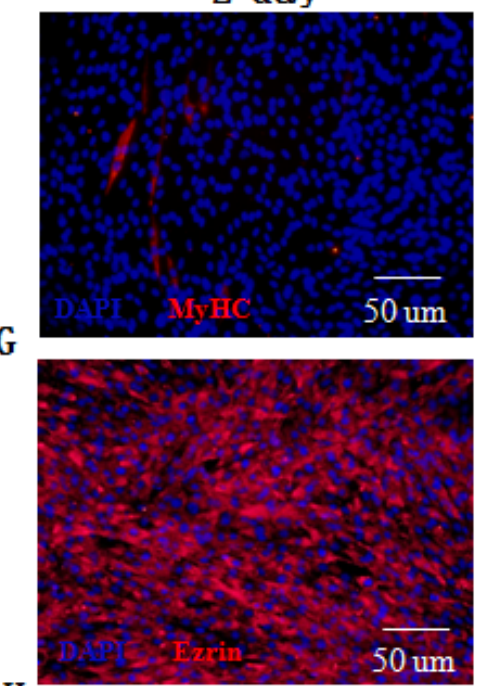

B DAPI

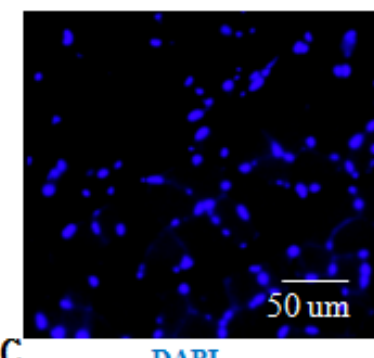

DAPI

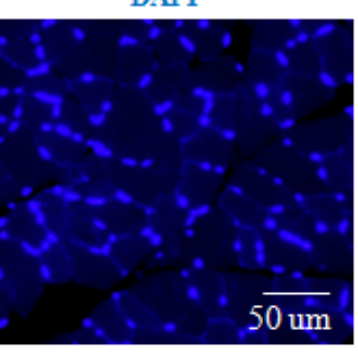

4 day
MyHC-I

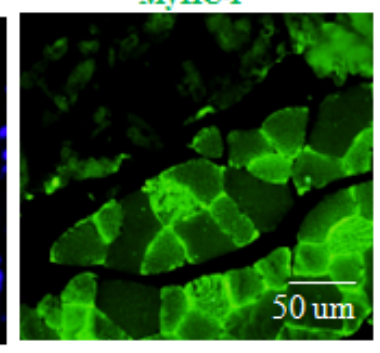

MyHC-II

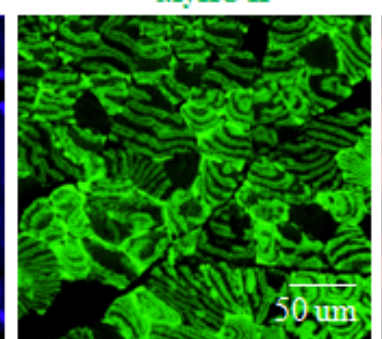

6 day

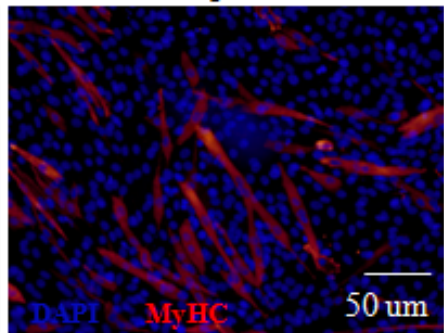

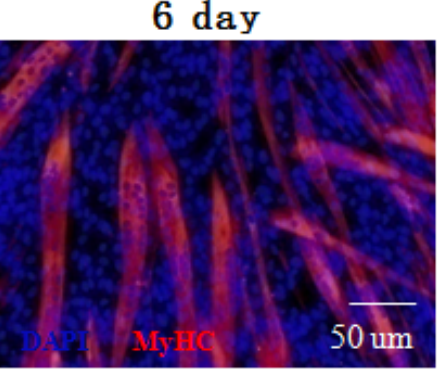
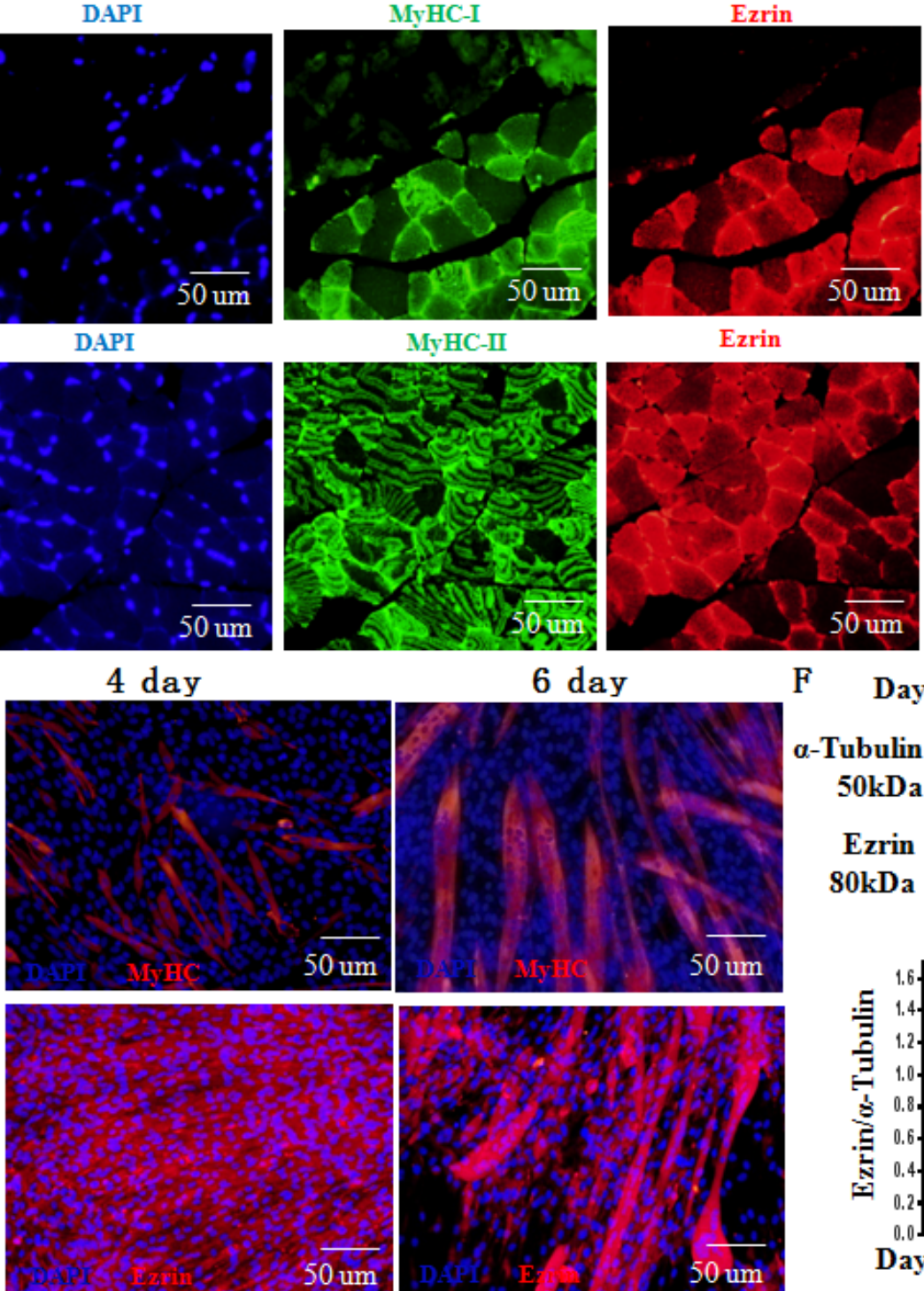

Ezrin

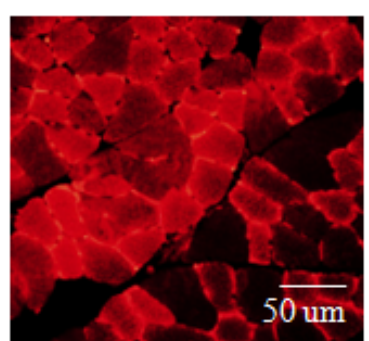

F

$\mathrm{H}$

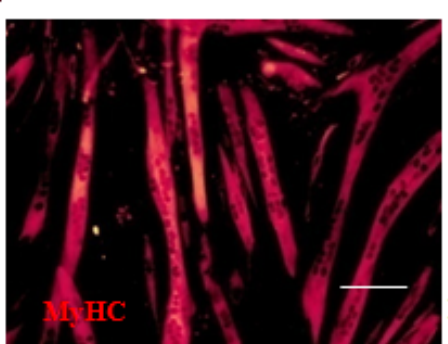

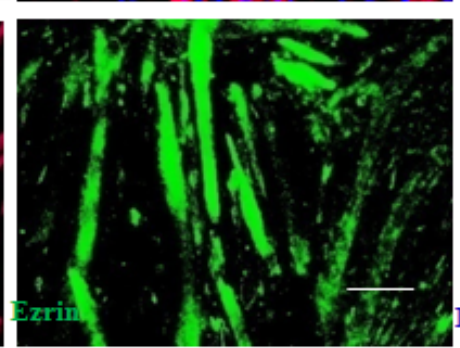

Day

a-Tubulin

$50 \mathrm{kDa}$

Ezrin
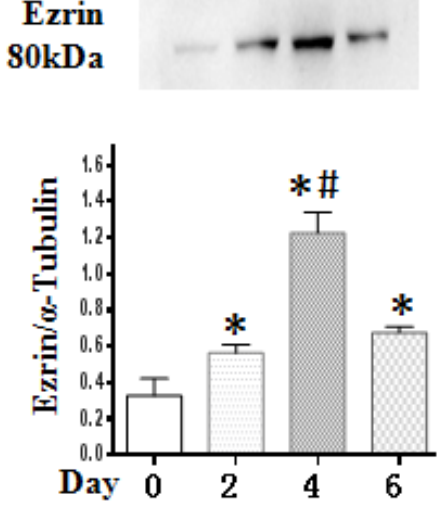

Merge

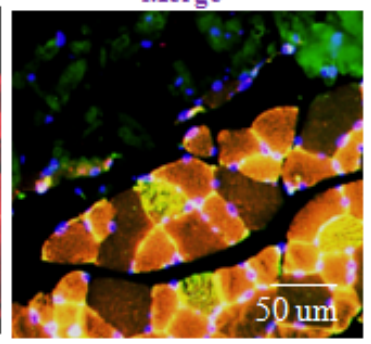

Merge
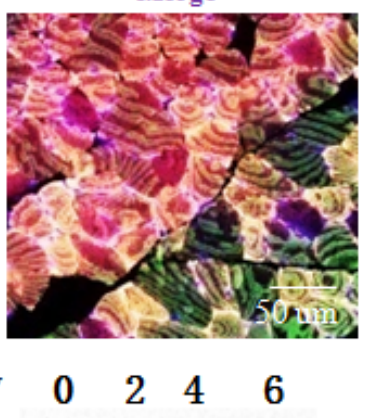

$\operatorname{mos}$

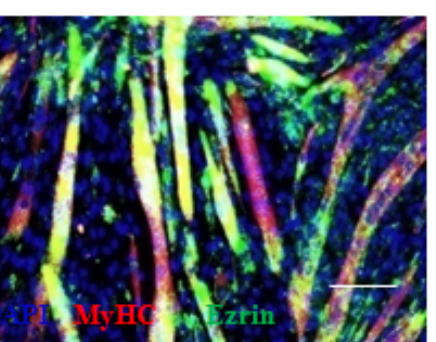




\section{Figure 1}

Ezrin was specifically expressed in myoblasts and their skeletal muscle fibers (A) Typical image for Ezrin expression in gastrocnemius muscle. Red fluorescent indicated Ezrin, DAPI indicated nucleus. (B) Typical image for Ezrin expression in soleus muscles. Red fluorescent indicated Ezrin; green fluorescent indicated MyHC-I; DAPI indicated nucleus. (C) Typical image for Ezrin expression in gastrocnemius muscle. Red fluorescent indicated Ezrin; green fluorescent indicated MyHC-I; DAPI indicated nucleus. (D) Quantitative assay for Ezrin expressions in muscle. (E) C2C12 myoblast differentiation model was established. Red fluorescent indicated MyHC; DAPI indicated nucleus. (F) Ezrin expressions were detected by Western blot at 0, 2, 4 and 6 days after myoblast differentiation. Quantitative assay for Ezrin expressions was analyzed 2, 4 and 6 days after myoblast differentiation. Three independent experiments were performed, $n=3$, *Pख0.05 vs. 0 day; \#P $₫ 0.05$ vs. 2 day; \&Pख0.05 vs. 4 day. (H) Typical image for co-location expression of MyHC and Ezrin at 6 days after myoblast differentiation. 
A
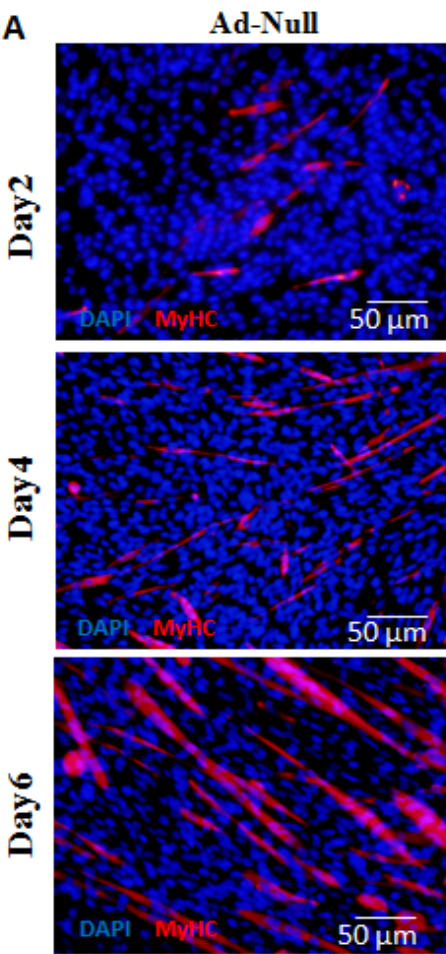

E
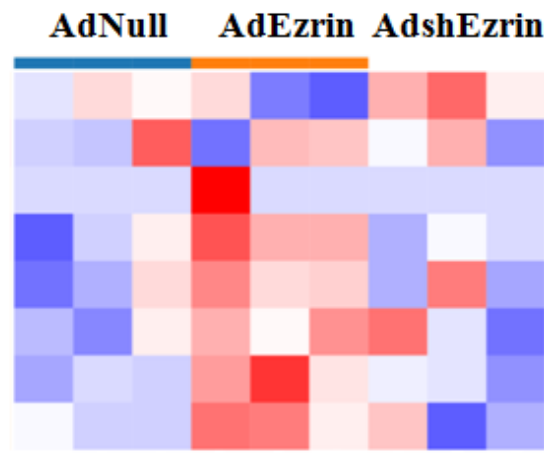

H

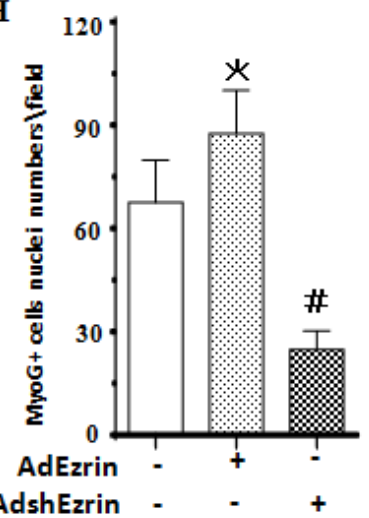

Ad-Ezrin
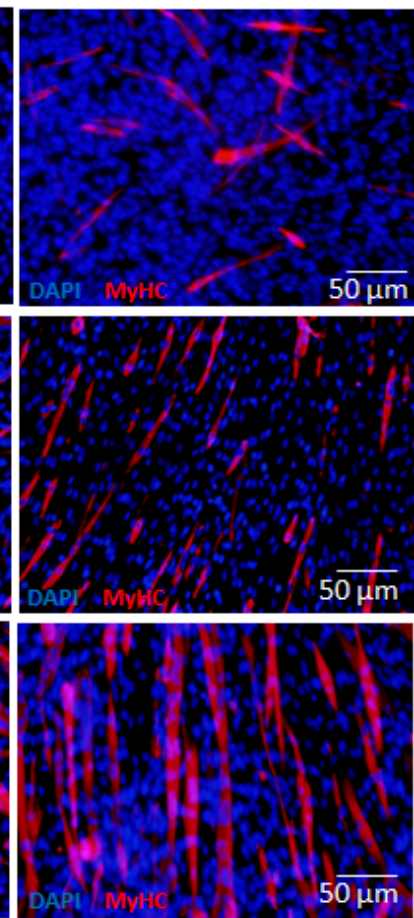

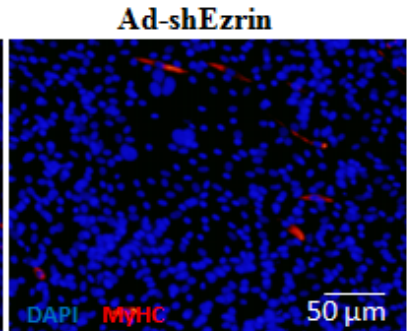

B
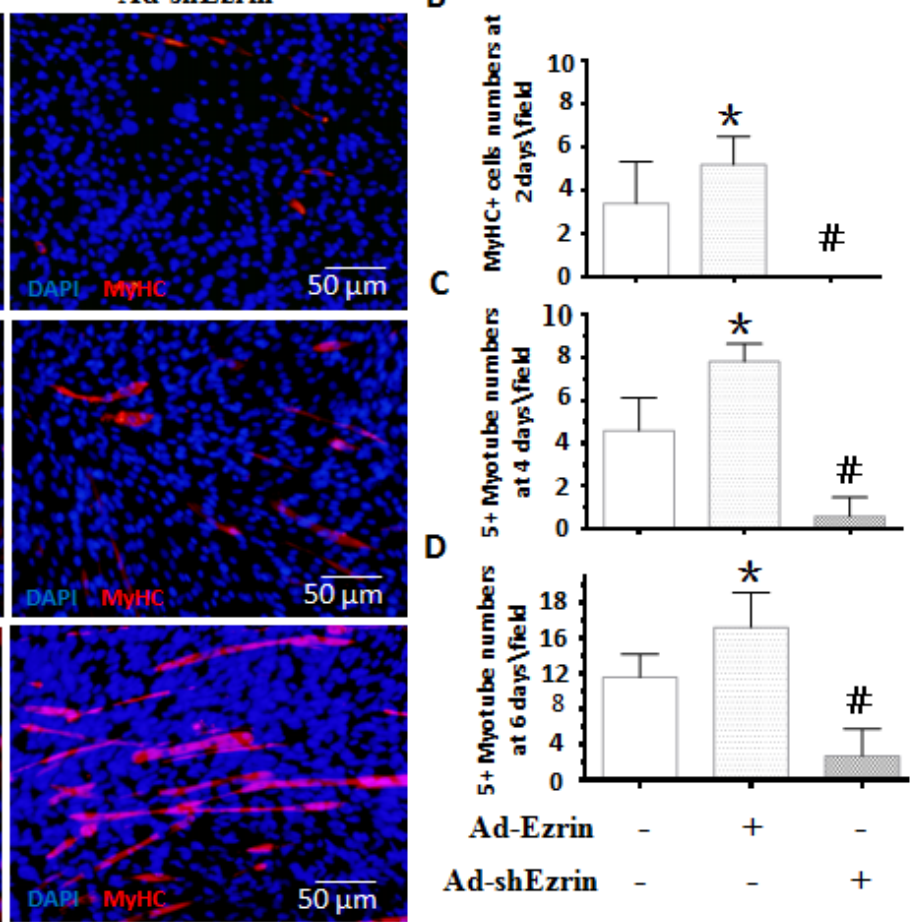

D

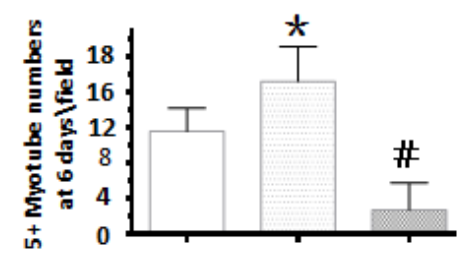

F

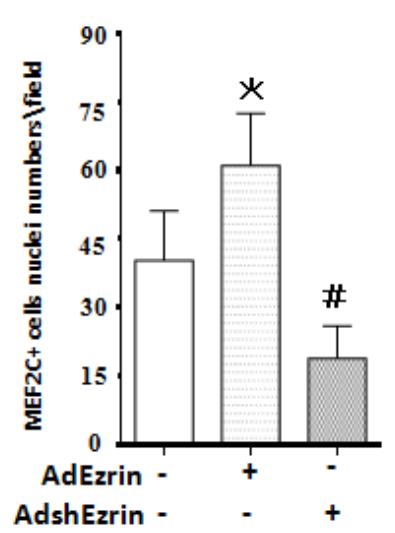

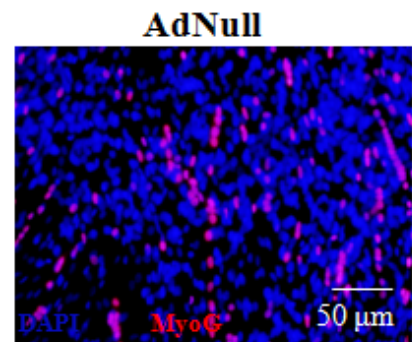

AdEzrin

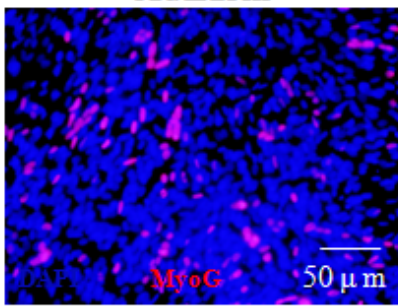

AdshEzrin

Ad-Ezrin

Ad-shEzrin - $\quad$ - $\quad+$

G

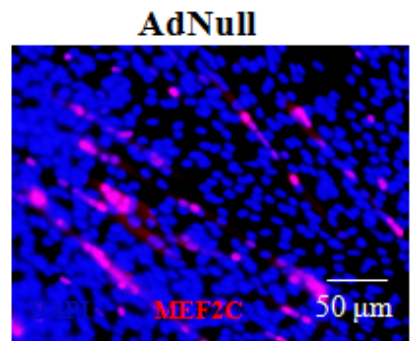

AdEzrin

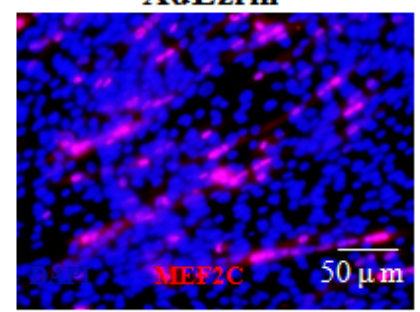

AdshEzrin
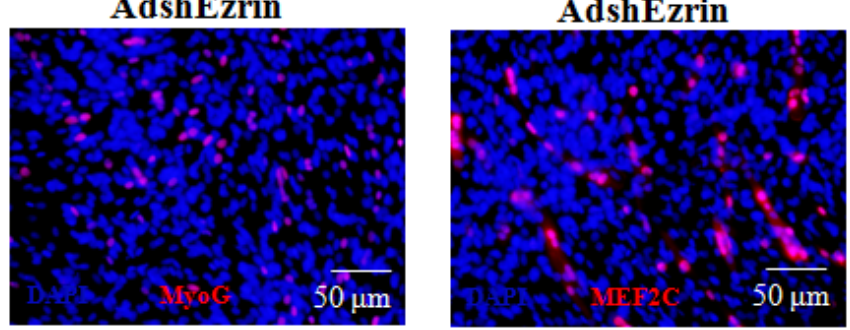

Figure 2

Ezrin involved in myoblast differentiation and fusion (A)Typical image of MyHC staining in differentiated C2C12 myoblast treated by overexpression or knockdown of Ezrin for 2, 4 and 6 days. (B-D) Quantitative assay for the number of MyHC+ myotubes with more than 5 nuclei was analyzed 2, 4 and 6 days after myoblast differentiation treated by overexpression or knockdown of Ezrin, respectively. Three independent experiments were performed, $n=3, * P \otimes 0.05$ vs. Ad-Null; \#Pख0.05 vs. Ad-Null. (E) Microarray 
analysis for myoblast differentiation in C2C12 myoblast treated by overexpression or knockdown of Ezrin at 6 days. $(\mathrm{H}-\mathrm{I})$ MyoG and MEF2c fluorescence staining and quantitative assay for myoblast differentiation. Red fluorescent indicated MyoG or MEF2c; DAPI indicated nucleus. Three independent experiments were performed, n=3, *Pख0.05 vs. Ad-Null; \#P $₫ 0.05$ vs. Ad-Null.

\section{Figure3.Ezrin involved myofiber specialization}

A

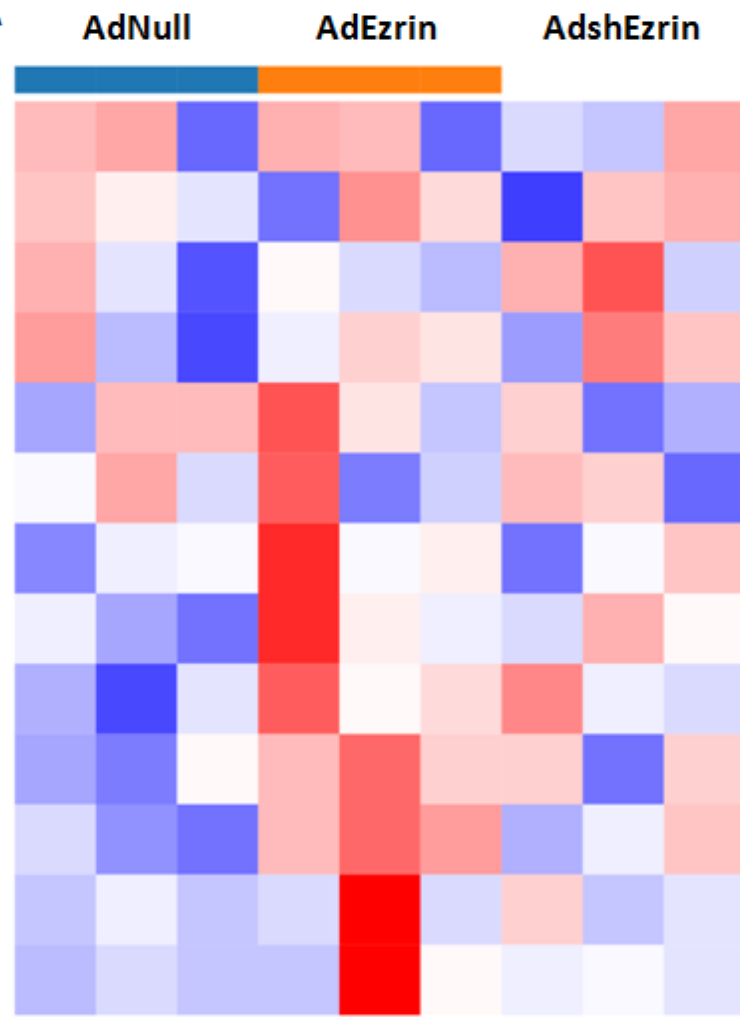

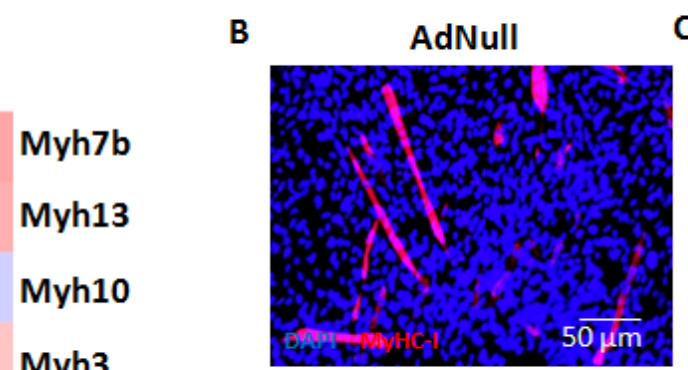

Myh3

MyHC-2X/D

Myh9

Myh10

Myh6

MyHC-1

MyHC-2A

MyHC-2B

Myh11

AdEzrin

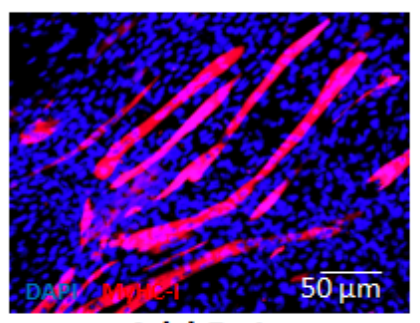

AdshEzrin

D myoblast fusion numbers $\geq 5$

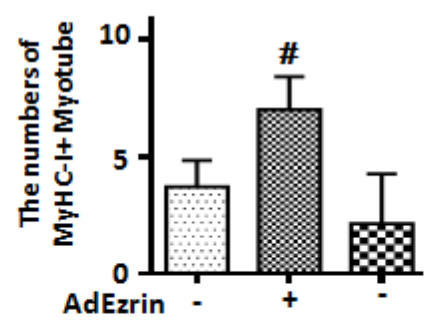

E
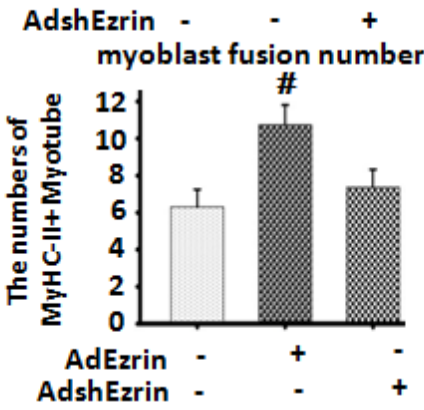

Ad-Null

$\Im$

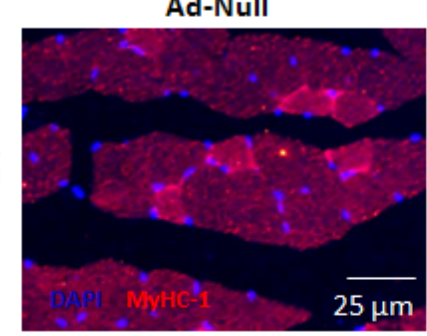

Ad-Null

H

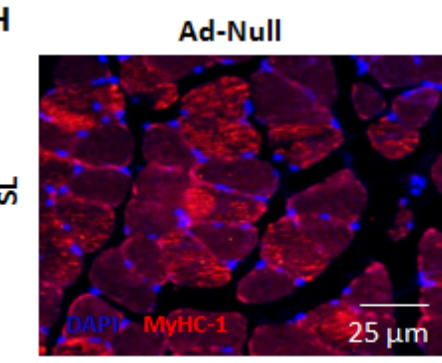

C
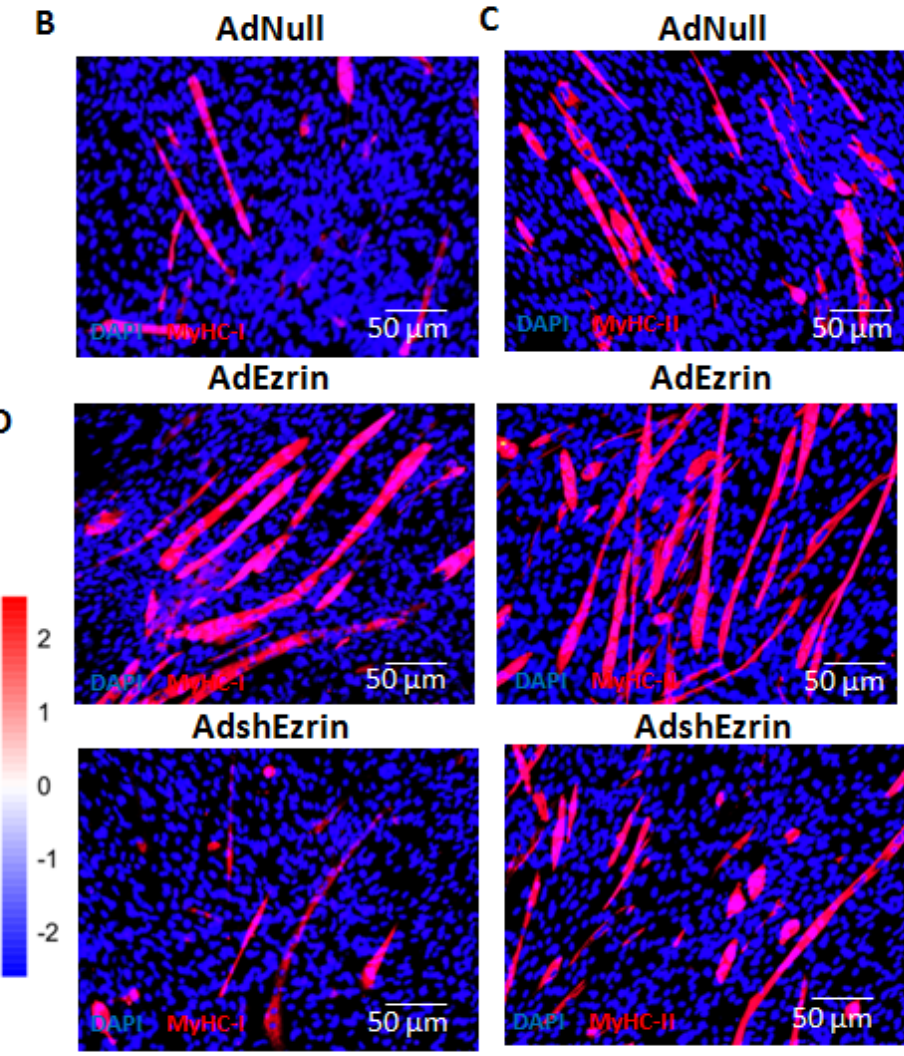

AdshEzrin

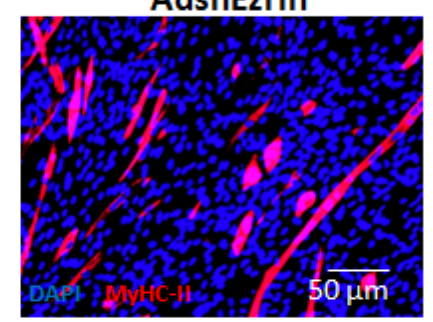

G
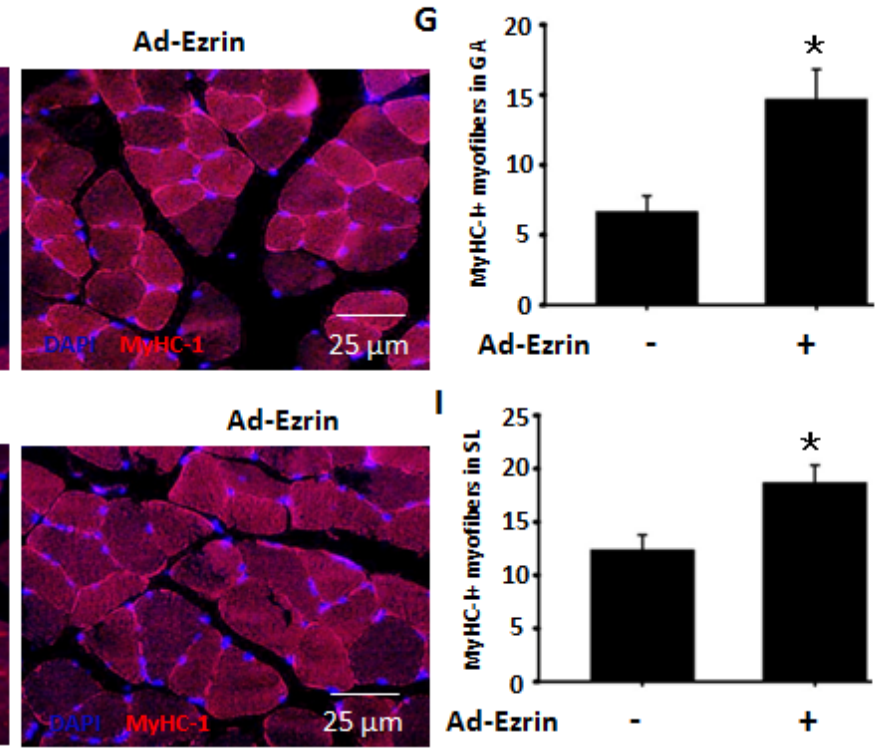

Figure 3

Ezrin involved myofiber specialization (A) Microarray analysis for myofiber types in C2C12 myoblast treated by overexpression or knockdown of Ezrin at 6 days. (B-E) MyHC-I and MyHC-II fluorescence 
staining (B-C) and quantitative assay (D-E) for myoblast differentiation. Red fluorescent indicated MyHC-I (B, NOQ) or MyHC-II (C, MY32); DAPI indicated nucleus. Three independent experiments were performed, $\mathrm{n}=3, * \mathrm{P} \otimes 0.05$ vs. Ad-Null; \#Pख0.05 vs. Ad-Null. (F-I) In vivo assay for MyHC-1 expressions in gastrocnemius $(\mathrm{GA})$ and soleus (SL) muscles following the local injection of Ad-Ezrin. Red fluorescent indicated MyHC-I (B, NOQ); DAPI indicated nucleus. $n=6, * P \llbracket 0.05$ vs. Ad-Null.

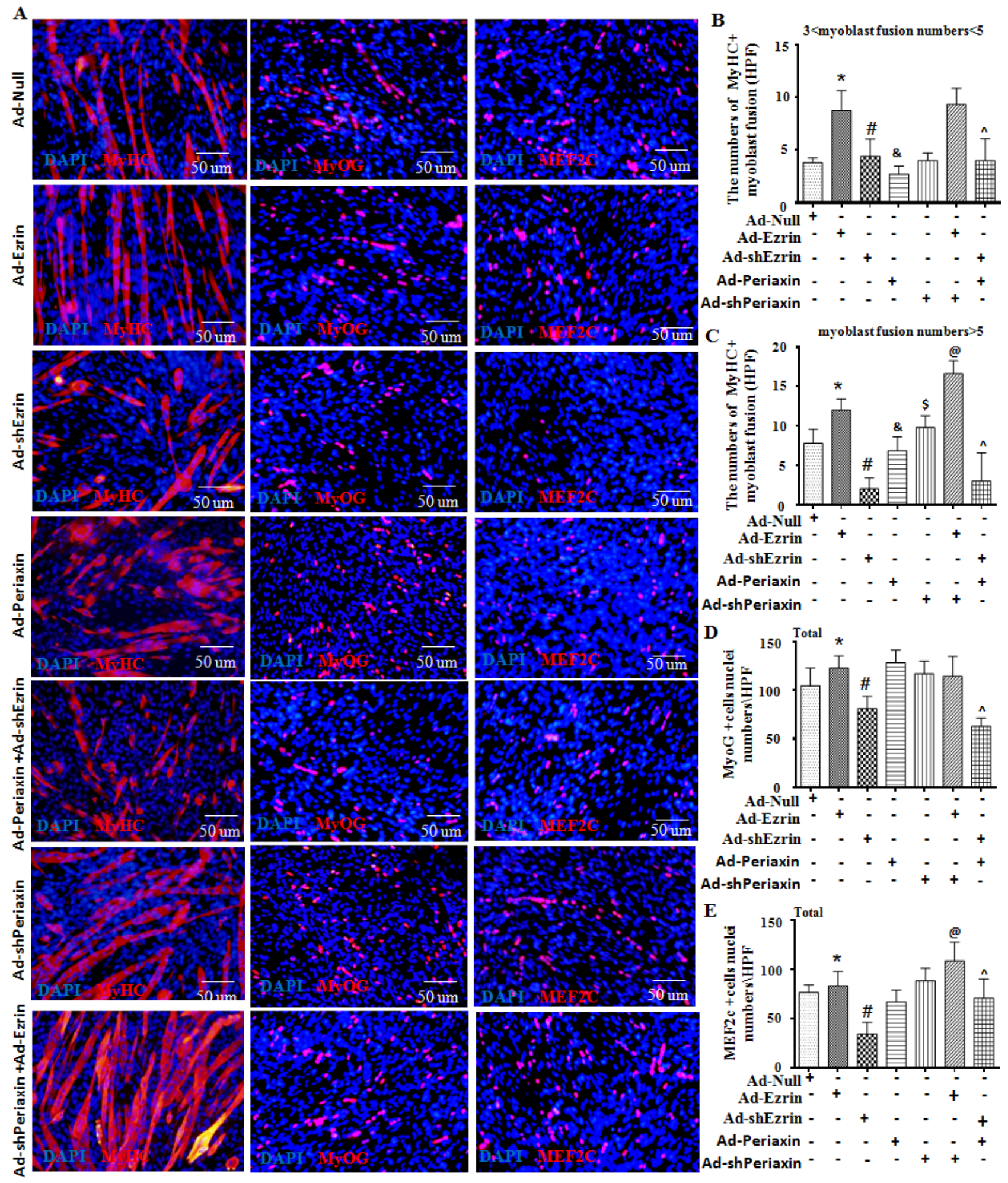

Figure 4 
Ezrin involved myoblast differentiation in L-Periaxin-dependent manner (A) Typical image of MyHC, MyoG or MEF2c staining in differentiated $\mathrm{C} 2 \mathrm{C} 12$ myoblast treated by overexpression or knockdown of Ezrin with or without Ad-Ad-Periaxin or Ad-shPeriaxin at 6 days of differentiation. Red fluorescent indicated MyHC, MyoG or MEF2C; DAPI indicated nucleus. (B-E) Quantitative assay for MyHC+ myotubes and $\mathrm{MEF} 2 \mathrm{c}+$ or MyoG + nuclei numbers in the above images. Three independent experiments were performed, $\mathrm{n}=3$, *Pख0.05 vs. Ad-Null; \#Pख0.05 vs. Ad-Null; \&Pख0.05 vs. Ad-Null; \$P冈0.05 vs. Ad-Null; @Pख0.05 vs. AdEzrin; ${ }^{\wedge} \mathrm{P} \otimes 0.05$ vs. Ad-shEzrin.
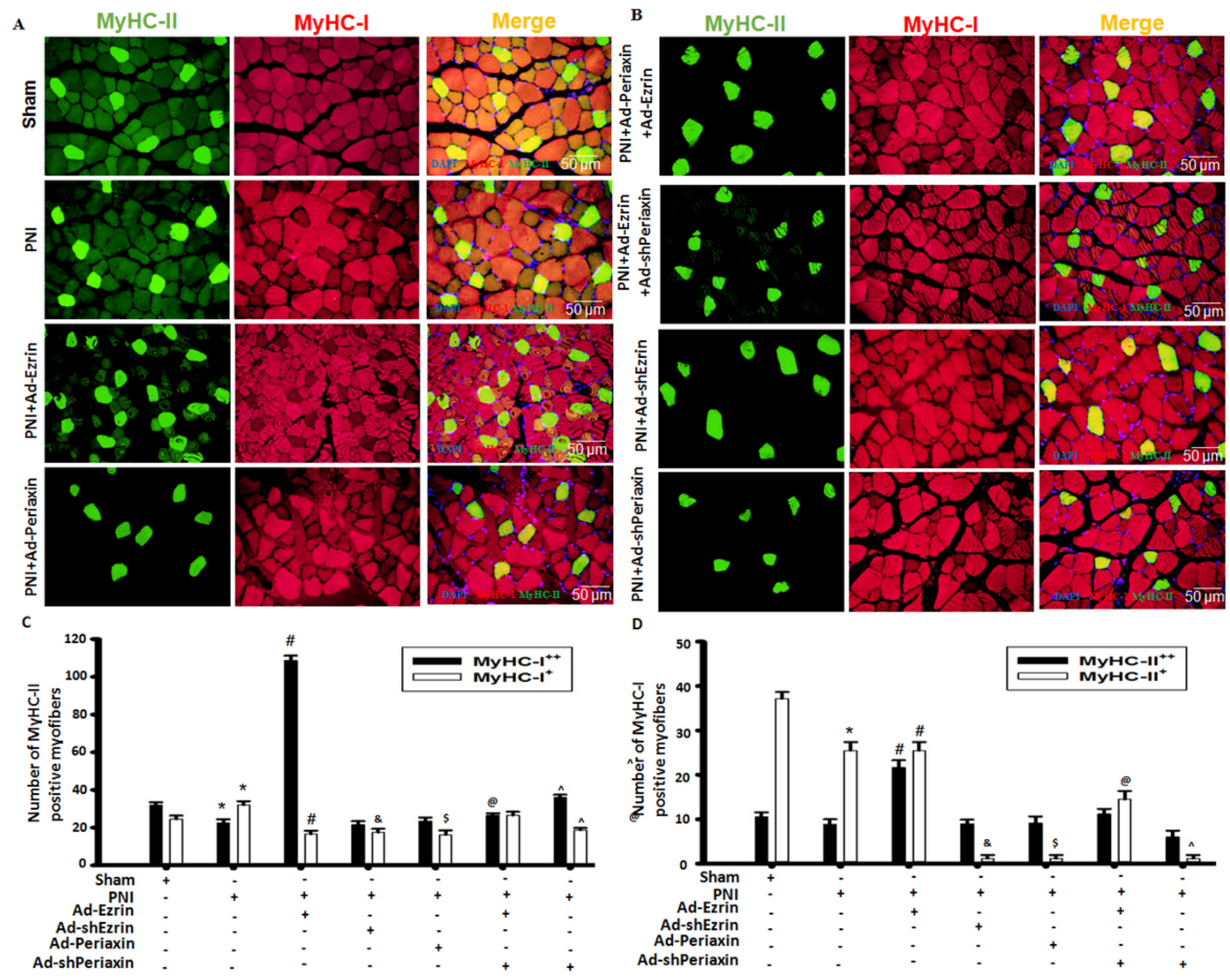

Figure 5

Ezrin involved in myofiber specialization in peroneal nerve injury in L-Periaxin-dependent manner (A)Typical image of MyHC-I (NOQ) or MyHC-II (MY32) staining in gastrocnemius muscle in peroneal nerve injury (PNI) model. Red fluorescent indicated MyHC-l; green fluorescent indicated MyHC-Il; DAPI indicated nucleus. (B-C) Quantitative assay for MyHC-I strong positive (MyHC-I++), MyHC-I weekly positive (MyHCI+), MyHC-Il strong positive (MyHC-II++) and MyHC-II weekly positive (MyHC-II+) myofibers in the above images, respectively. Red fluorescent indicated MyHC-l; green fluorescent indicated MyHC-II; DAPI 


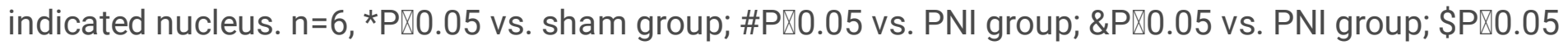
vs. PNI group; @P®0.05 vs. Ad-Ezrin; ^P®0.05 vs. PNI group.
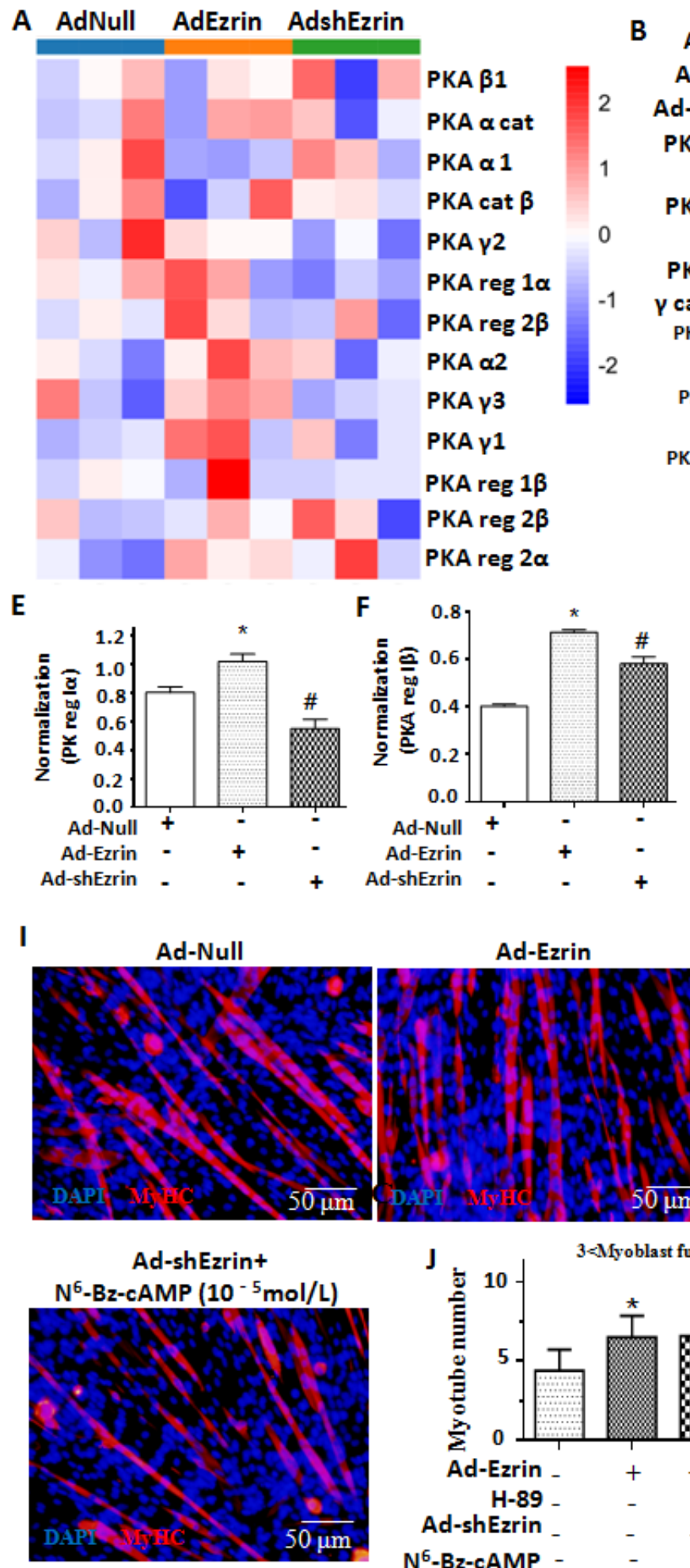
Ad-Ezrin
B Ad-Null Ad-Ezrin Ad-shEzrin PKA $y$ cat $40 \mathrm{kDa}$

PKA $\alpha$ cat $40 \mathrm{kDa}$

PKA $\alpha / \beta /$ $\gamma$ cat $40 \mathrm{kDa}$ PKAreg I $\alpha$ 43 kDa $51 \mathrm{kDa}$ PKAreg I I $\alpha$ $50 \mathrm{kDa}$ GAPDH $36 \mathrm{kDa}$

G
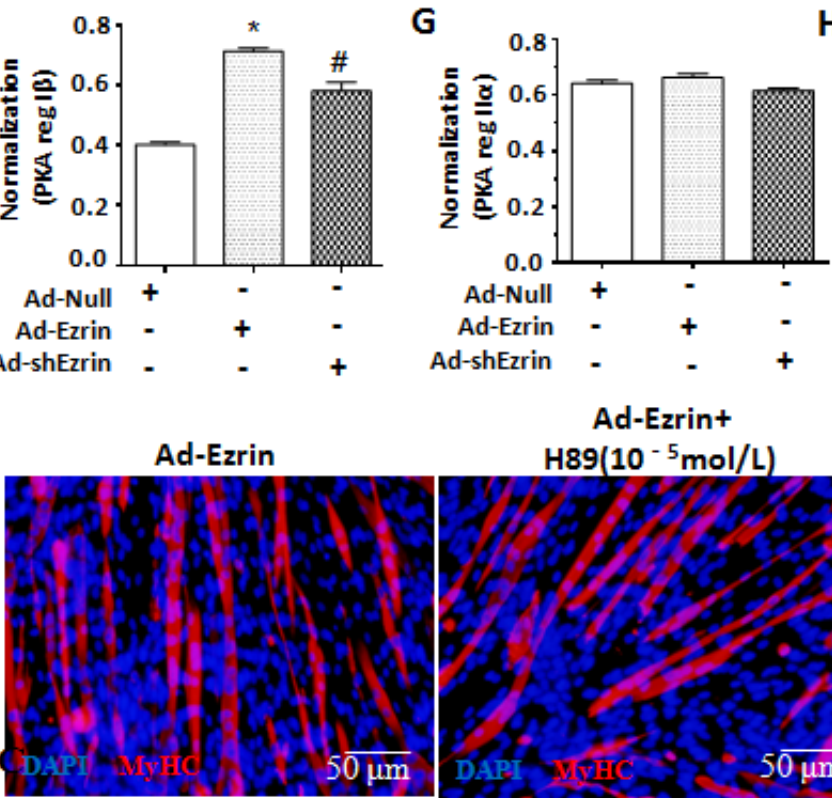

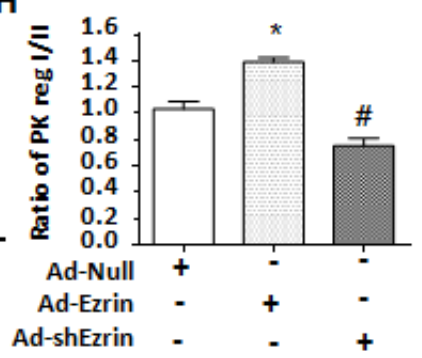

Ad-Ezrint
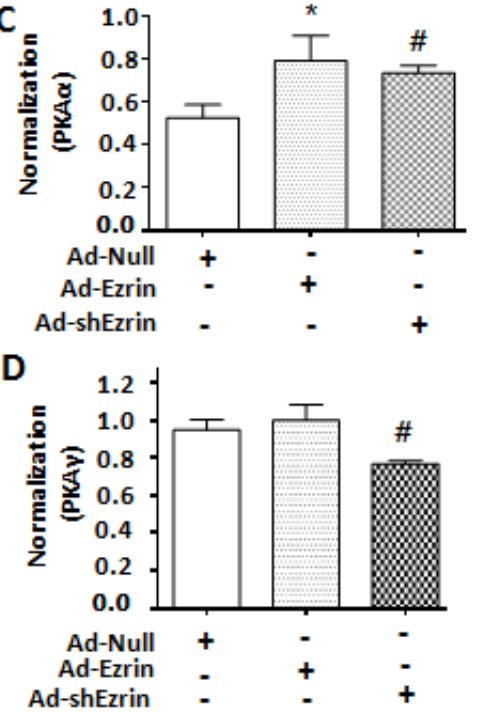

H H89 $\left(10^{-5} \mathrm{~mol} / \mathrm{L}\right)$
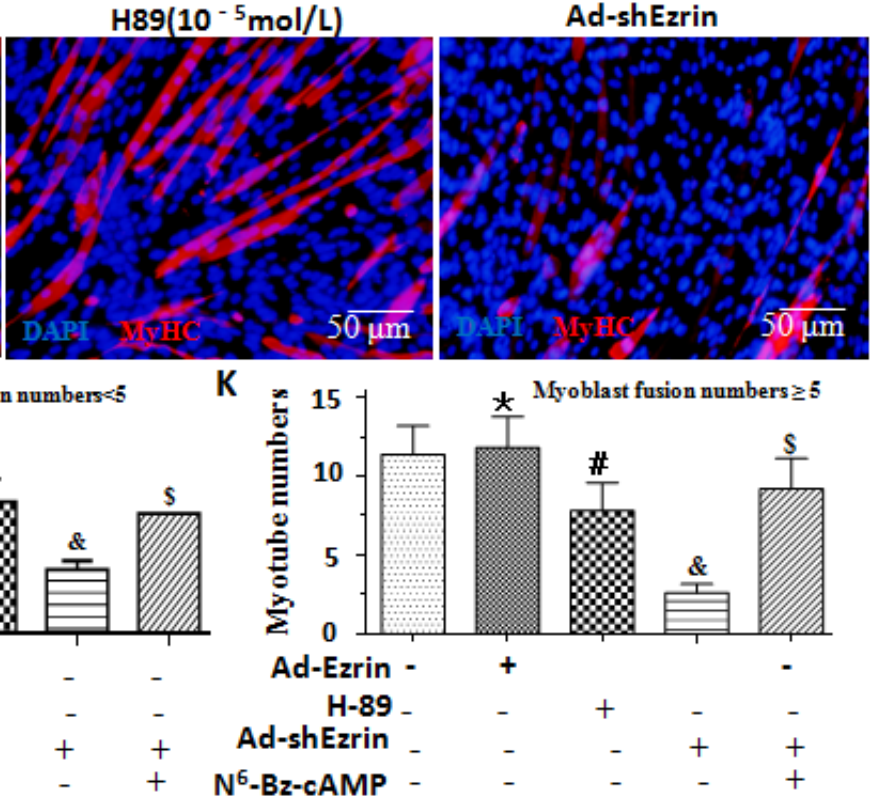

Figure 6

Ezrin regulated myoblast differentiation and fusion through PKA signaling pathway (A) Microarray analysis for PKA signaling pathway in C2C12 myoblast treated by overexpression or knockdown of Ezrin at 6 days. (B) Western blot for indicated proteins in myoblast treated by over-expressionor knockdown of 
Ezrin for 6 days. (C-H) Quantitative assay for indicated proteins was analyzed 6 days after myoblast differentiation following the treatment of over-expression or knockdown of Ezrin. Three independent experiments were performed, $n=3$, *Pख0.05 vs. Ad-Null; \#Pख0.05 vs. Ad-Null. (I) Typical image of MyHC staining in differentiated $\mathrm{C} 2 \mathrm{C} 12$ myoblast treated by Ad-Ezrin with or without $\mathrm{H}-89$ (10-5mol/L), or AdshEzrin with or without N6-Bz-cAMP (10-5mol/L). Red fluorescent indicated MyHC; DAPI indicated nucleus. (J-K) Quantitative assay for the number of MyHC+ myotubes with 3-5 or more than 5 nuclei were analyzed six days after myoblast differentiation. Three independent experiments were performed, $n=3$, * $P$ $\llbracket 0.05$ vs. Ad-Null; \#Pख0.05 vs. Ad-Ezrin; \&Pख0.05 vs. Ad-Null; \$P『0.05 vs. Ad-shEzrin. 


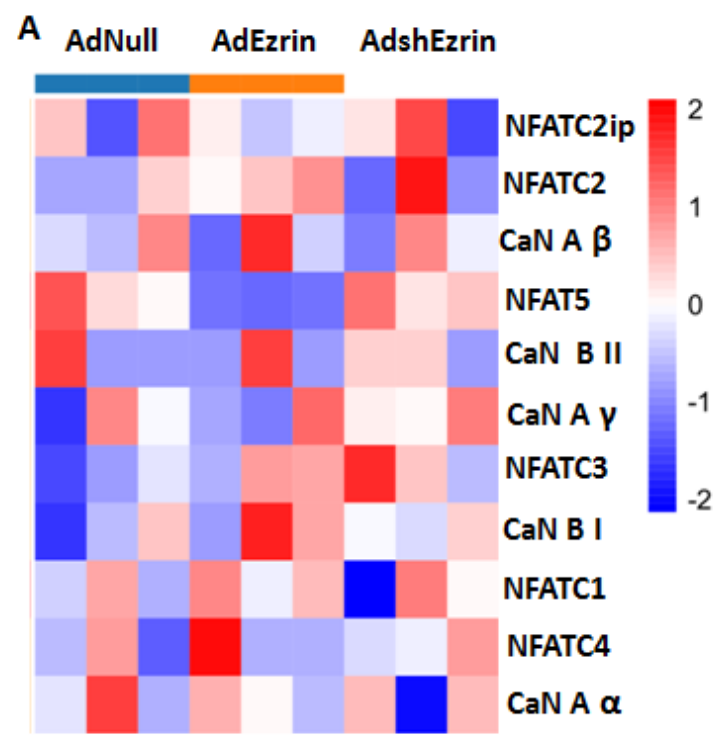

B

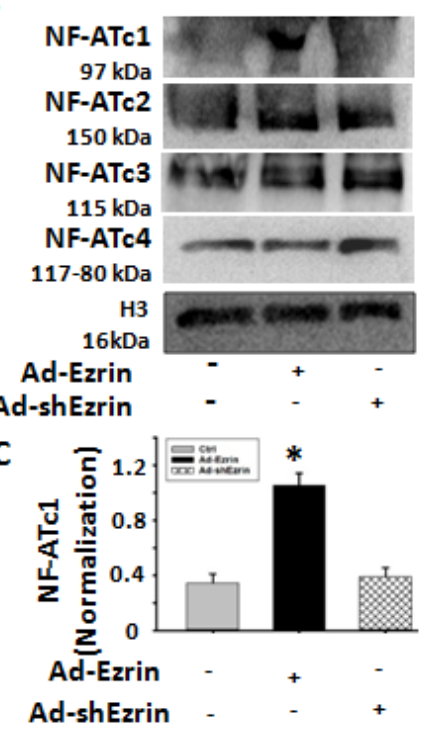

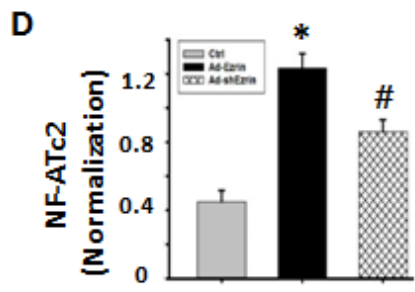

Ad-Ezrin

Ad-shEzrin

E

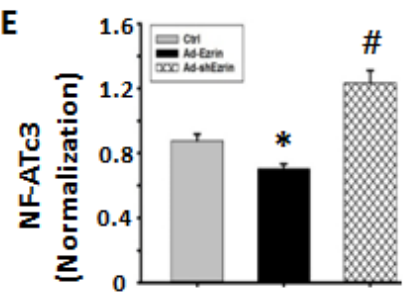

Ad-Ezrin

Ad-shEzrin
$\mathbf{F}$
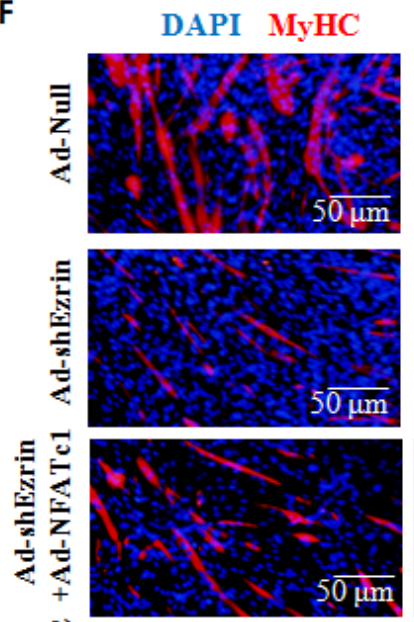

卷
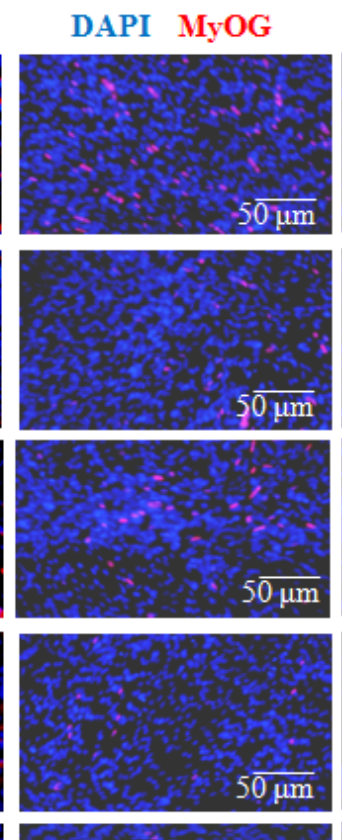

管

焉焉
DAPI MEF2C
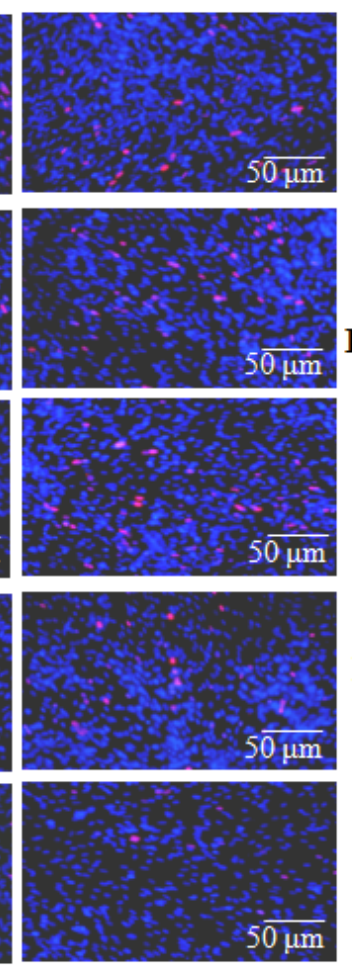

G
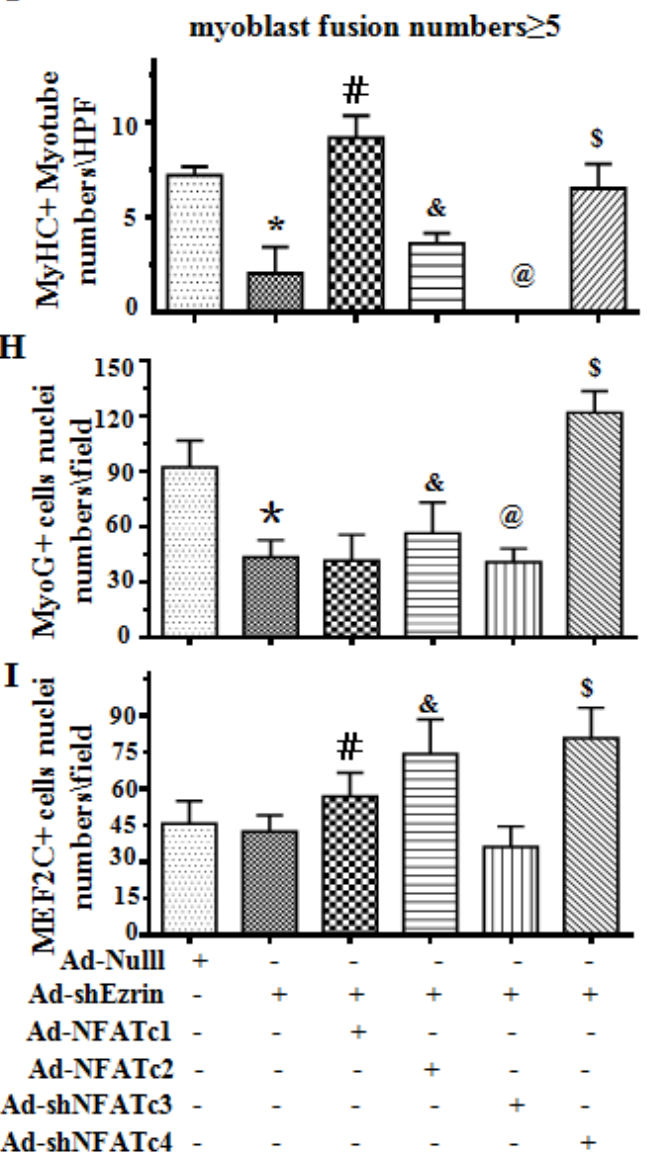

\section{Figure 7}

NFAT involved in Ezrin-mediated myoblast differentiation and fusion (B) Microarray analysis for CaNNFAT signaling pathway in $\mathrm{C} 2 \mathrm{C} 12$ myoblast treated by overexpression or knockdown of Ezrin at 6 days. (B) Western blot of NFATc1-c4 proteins in myoblast treated by over-expression or knockdown of Ezrin for 6 days. (C-E) Quantitative assay of NFATc1-c4 proteins was analyzed 6 days after myoblast differentiation following the treatment of over-expression or knockdown of Ezrin. Three independent 
experiments were performed, n=3, *P囚0.05 vs. Ad-Null; \#P囚0.05 vs. Ad-Null. (F) Typical image of MyHC, MyoG or MEF2c staining in differentiated C2C12 myoblast treated by Ad-shEzrin with or without Ad-

NFATc1, Ad-NFATc2, Ad-shNFATc3 and Ad-shNFATc4. Red fluorescent indicated MyHC, MyoG or MEF2c, respectively; DAPI indicated nucleus. (G-I) Quantitative assay for MyHC+ myotubes and MEF2c+ or

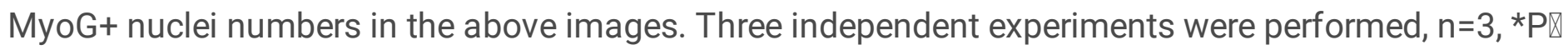

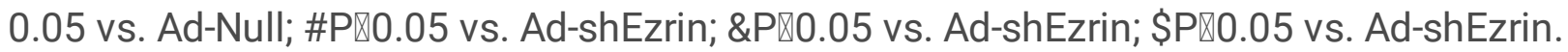
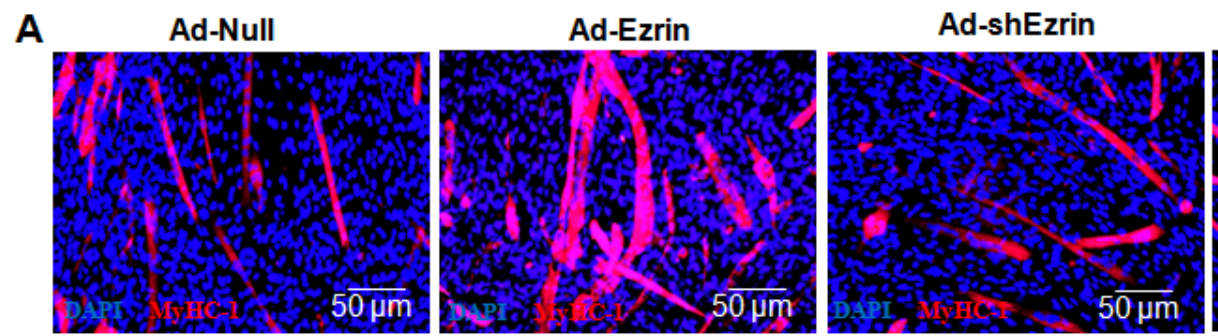

Ad-shEzrin+Ad-NFATc1
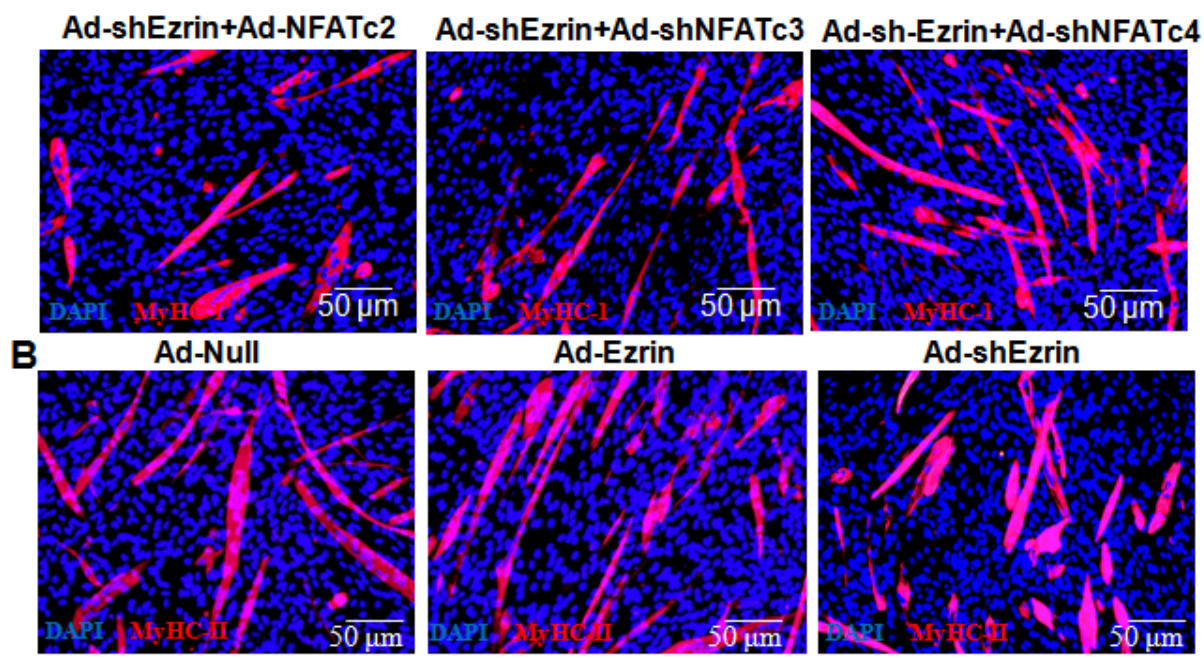

Ad-shEzrin+Ad-NFATc1
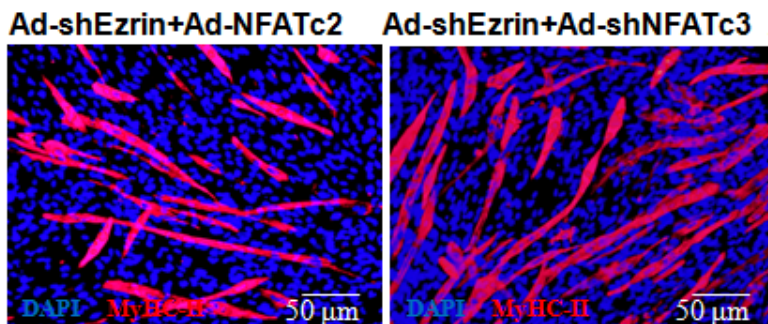

C

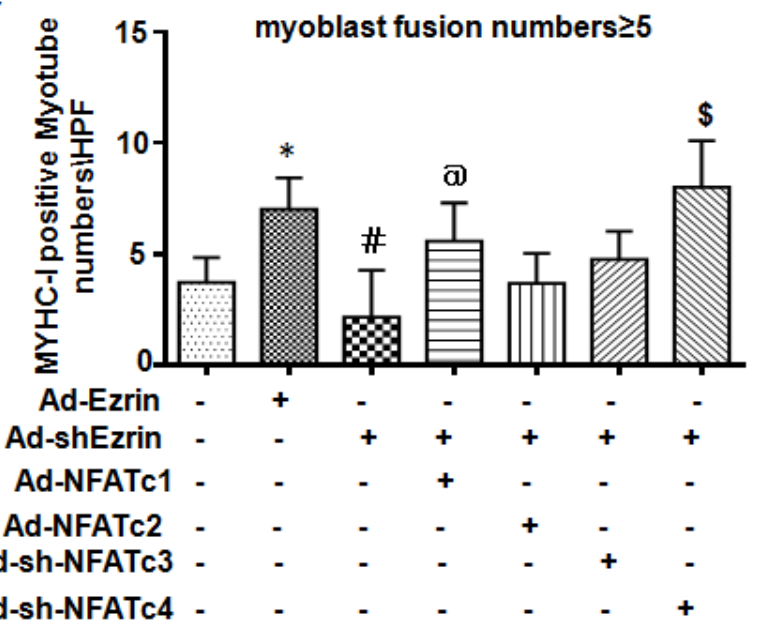

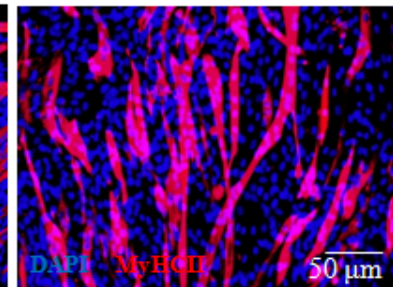

D

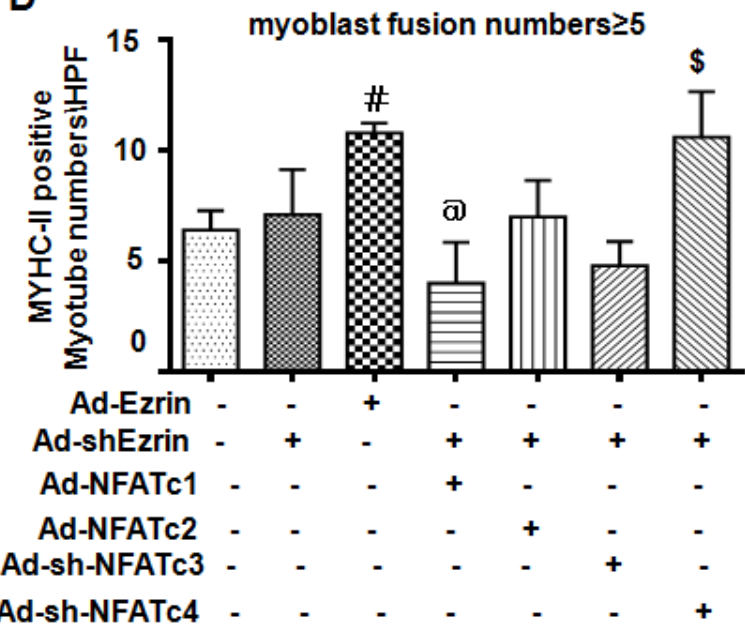

Figure 8 
Ezrin regulated myofiber specialization through NFATs signaling pathway (A-B) Typical image of MyHC-I (NOQ) or MyHC-II (MY32) staining in in differentiated C2C12 myoblast treated by Ad-shEzrin with or without Ad-NFATc1, Ad-NFATc2, Ad-shNFATc3 and Ad-shNFATc4. Red fluorescent indicated MyHC-I (A) or MyHC-II (B); DAPI indicated nucleus. (C-D) Quantitative assay for MyHC-I positive, MyHC-Il positive myotubes in the above images, respectively. Three independent experiments were performed, $n=3, * P \rrbracket$

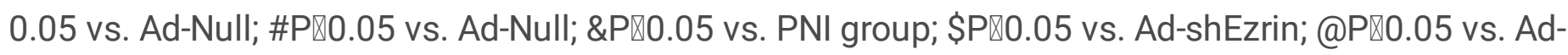
shEzrin.

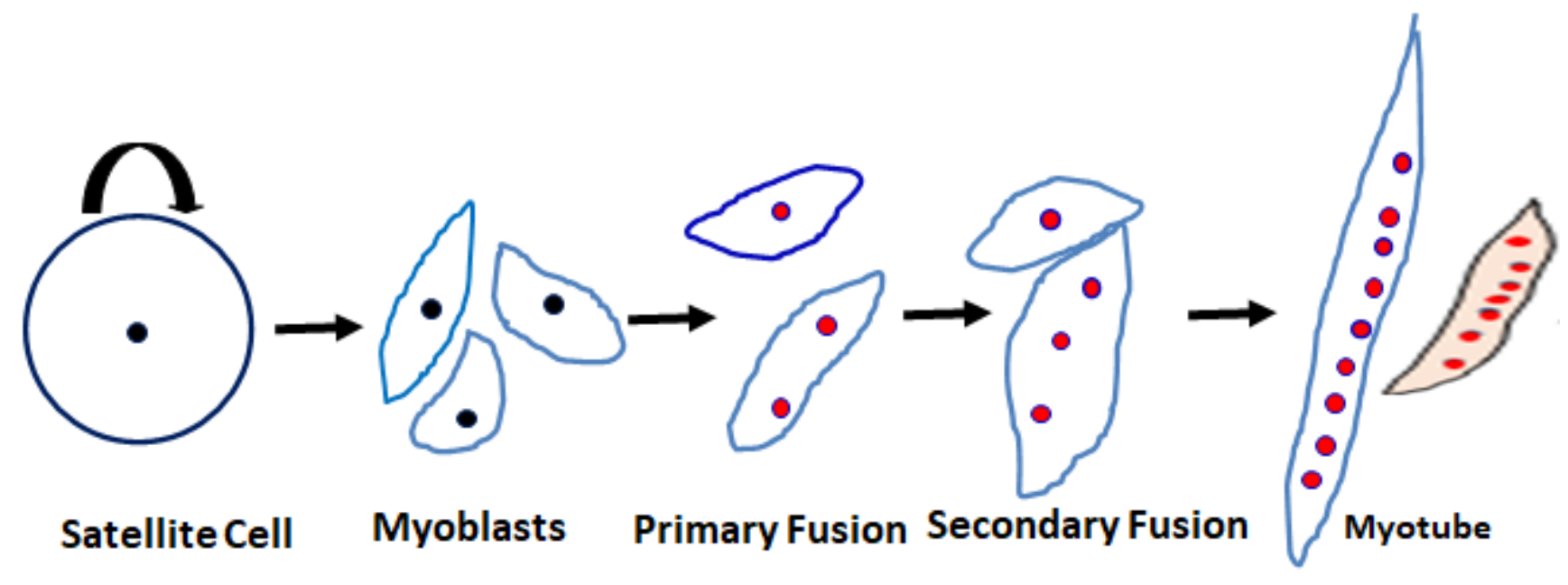

Ezrin Periaxin

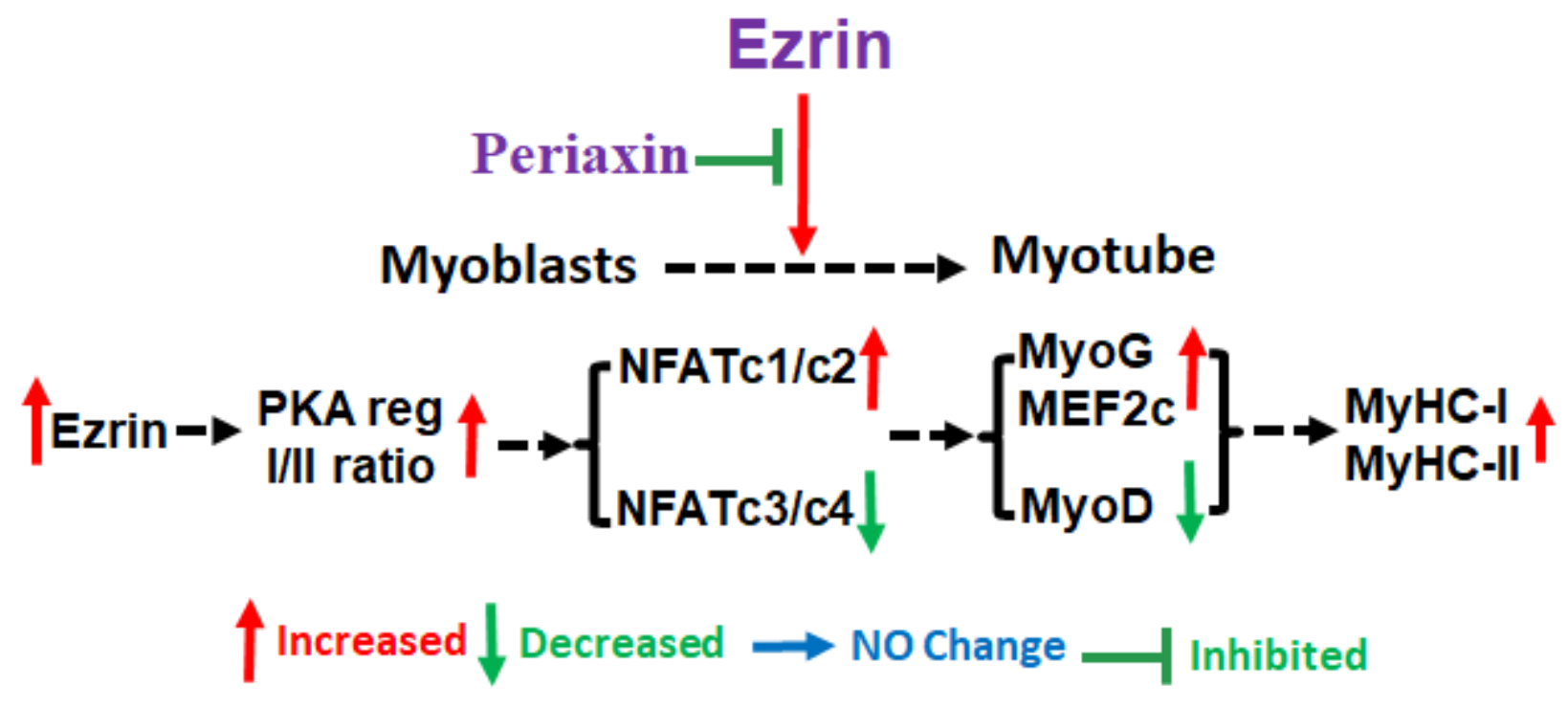

Figure 9

Working Model: Ezrin prompted myoblast differentiation and muscle fiber specialization and gastrocnemius muscle repair in peroneal nerve injury model through PKA-NFAT-MyoG/MEF2csignaling pathway Ezrin was expressed in $\mathrm{MyHC} \mathrm{I/II} \mathrm{myofibers} \mathrm{in} \mathrm{vivo,} \mathrm{and} \mathrm{time-dependently} \mathrm{increased} \mathrm{during}$ myoblast differentiation/fusion in vitro. Interaction between Ezrin and Periaxin control myoblast 
differentiation/fusion. Ezrin activated PKA-NFAT-MEF2C signaling pathway, triggering myoblast differentiation/fusion and muscle fiber specialization.

\section{Supplementary Files}

This is a list of supplementary files associated with this preprint. Click to download.

- sFigure1.tif

- sFigure2.tif

- sFlgure3.tif

- sFigure4.tif

- sFigure5.tif

- sFigure6.tif

- sFigure7.tif

- sFigure8.tif

- sFigure9.tif

- sFigure10.tif 